University of Massachusetts Amherst

ScholarWorks@UMass Amherst

\title{
Natural implementation with partially-honest agents in economic environments with free-disposal
}

\author{
Michele Lombardi \\ University of Glasgow \\ Naoki Yoshihara \\ The Institute of Economic Research, Hitotsubashi University
}

Follow this and additional works at: https://scholarworks.umass.edu/econ_workingpaper

Part of the Economics Commons

\section{Recommended Citation}

Lombardi, Michele and Yoshihara, Naoki, "Natural implementation with partially-honest agents in economic environments with free-disposal" (2014). Economics Department Working Paper Series. 175. https://doi.org/10.7275/6356180

This Article is brought to you for free and open access by the Economics at ScholarWorks@UMass Amherst. It has been accepted for inclusion in Economics Department Working Paper Series by an authorized administrator of ScholarWorks@UMass Amherst. For more information, please contact scholarworks@library.umass.edu. 


\section{DEPARTMENT OF ECONOMICS}

Working Paper

Natural implementation with partially-honest agents in economic environments with freedisposal

by

Lombardi, Michele Yoshihara, Naoki

UNIVERSITY OF MASSACHUSETTS AMHERST 


\title{
Natural implementation with partially-honest agents in economic environments with free-disposal ${ }^{*}$
}

\author{
Michele Lombardi ${ }^{\dagger}$ \\ University of Glasgow \\ Naoki Yoshihara ${ }^{\ddagger}$ \\ Hitotsubashi University, IER
}

September 4, 2014

\begin{abstract}
We study Nash implementation by natural price-quantity mechanisms in pure exchange economies with free-disposal (Saijo et al., 1996, 1999) where agents have weak/strong intrinsic preferences for honesty (Dutta and Sen, 2012). Firstly, the Walrasian rule is shown to be non-implementable where all agents have weak (but not strong) intrinsic preferences for honesty. Secondly, the class of efficient allocation rules that are implementable is identified provided that at least one agent has strong intrinsic preferences for honesty. Lastly, the Walrasian rule is shown to belong to that class.
\end{abstract}

JEL classification: C72; D71.

Key-words: Natural implementation, Nash equilibrium, exchange economies, intrinsic preferences for honesty.

*We are grateful to Bram Driesen, Hitoshi Matsushima, Hervé Moulin, Hans Peters, Tatsuyoshi Saijo, Arunava Sen, David Wettstein, Nicholas C. Yannelis, for useful comments and suggestions. Special thanks go to exceptionally thorough referees and an advisory editor, whose comments and suggestions have led to substantial improvements in the paper. The usual caveat applies.

${ }^{\dagger}$ Adam Smith Business School, University of Glasgow, Glasgow, G12 8QQ, United Kingdom, e-mail: michele.lombardi@glasgow.ac.uk.

¥Institute of Economic Research, Hitotsubashi University, 2-4 Naka, Kunitachi, Tokyo, 186-8603 Japan, e-mail: yosihara@ier.hit-u.ac.jp. 


\section{Introduction}

In implementation problems in classical economic environments, as Hurwicz (1978), Otani and Sicilian (1982), and Thomson (1984) discuss, the information that agents exchange in the allocation process typically concerns ongoing prices and their optimal consumption bundles at these prices, that is, information that lies in the graph of agents' true demand correspondences. Therefore, a prominent and natural restriction on mechanisms for those problems would be represented by price-quantity mechanisms, where each agent's message consists of a pair of a price vector and a demand quantity. Moreover, to meet the requirements of an adequate theory of implementation (see, Jackson, 1992), Dutta et al. (1995), Sjöström (1996), and Saijo et al. (1996, 1999) define the class of natural mechanisms in economic environments, a typical example of which is a price-quantity mechanism satisfying feasibility, forthrightness, and the best response property; henceforth, natural price-quantity mechanism (natural $p-q$ mechanism).

In a seminal study that initiated a burgeoning literature, Matsushima (2008a, 2008b) and Dutta and Sen (2012) studied implementation problems where agents have intrinsic preferences for honesty. Loosely, an agent has intrinsic preferences for honesty - called partially-honest - when she strictly prefers to tell the truth whenever lying has no effect on her material payoff. ${ }^{1}$

This paper studies Nash implementation with partially-honest agents by natural $p-q$ mechanisms in pure exchange economies with free-disposal. ${ }^{2}$ It is built upon two alternative notions of intrinsic preferences for honesty, though for both of them the point of departure is that of the agent's true demand correspondence. With one type, a partially-honest agent has strong intrinsic preferences for honesty if her truthful report consists of true prices as well as of her true optimal bundle at those prices coinciding with the bundle recommended by the allocation rule for the underlying economy. With the other type, a partially-honest agent has weak intrinsic preferences for honesty if her truthful report consists of her true optimal bundle compatible with the allocation rule.

In a separable environment similar to ours, Dutta and Sen (2012) and Kartik et al. (2014) show that any social choice function $(S C F)$ is implementable by a mechanism without any "tail-chasing" construction where all

\footnotetext{
${ }^{1}$ There are seminal related works such as Glazer and Rubinstein (1998), Eliaz (2002), Corchón and Herrero (2004), and Kartik and Tercieux (2012).

${ }^{2}$ In economies with free-disposal the mechanism must only be weakly balanced.
} 
agents have small intrinsic preferences for honesty (a more detailed discussion is given in section 3$)^{3}$ and, like their results, none of ours hinge on any sort of "tail-chasing" construction. ${ }^{4}$ Similarly, we also observe in section 3 that, in light of the characterization result of Lombardi and Yoshihara (2013b), any efficient $S C F$ is implementable by a natural $p-q$ mechanism where all agents have weak intrinsic preferences for honesty. This general positive result crucially relies on the single-valuedness of allocation rules. If one abandons the latter requirement (in favor of multi-valued allocation rules), that result cannot be maintained. In fact, no natural price-quantity mechanism can implement the Walrasian rule where all agents have weak (but not strong) intrinsic preferences for honesty. ${ }^{5}$ Also, this contextualizing example provides rationales that support the study of the strong variation of honesty, which, in our view, is more appropriate for the type of implementation problems at hand as well as more intimately connected with the notion of market equilibrium. Then, in section 4 , we identify the class of efficient allocation rules that are implementable by natural $p-q$ mechanisms provided that there are partially-honest agents having strong intrinsic preferences for honesty. Also, the Walrasian rule is shown to belong to this class.

Section 2 of this paper presents the theoretical framework and outlines the basic model, whereas the main results are presented in sections 3 and 4 with a brief concluding section 5 .

\section{The model}

There are $n \geq 3$ agents in $N \equiv\{1, \ldots, n\}$ and $\ell \geq 2$ distinct commodities in $L \equiv\{1, \ldots, \ell\}$. $\mathbb{R}$ is the set of reals; $\mathbb{R}_{+}\left(\mathbb{R}_{++}\right)$denotes the set of nonnegative (positive) reals; $\mathbb{R}^{\ell}$ is the Cartesian product of ordered $\ell$-tuples of reals, whereas $\mathbb{R}_{+}^{\ell}\left(\mathbb{R}_{++}^{\ell}\right)$ denotes its non-negative (positive) orthant. Vector inequalities are defined as follows: For all $x, y \in \mathbb{R}^{\ell}, x \geq y$ if $x_{\ell} \geq y_{\ell}$ for each $\ell \in L, x>y$ if $x \geq y$ and $x \neq y$, and $x \gg y$ if $x_{\ell}>y_{\ell}$ for each $\ell \in L$.

\footnotetext{
${ }^{3}$ Their equilibrium solutions are not the Nash one, as discussed in section 3 below.

${ }^{4}$ Loosely speaking, the basic idea of the argument is that if agents announce a nonequilibrium message profile, the mechanism provides agents with material incentives to coordinate on an equilibrium message profile. This idea crucially hinges on the fact that agents have intrinsic preferences for honesty and that free-disposal is allowed.

${ }^{5}$ We thank an anonymous reviewer for drawing our attention to this point. Also recall that the Walrasian rule is not Nash implementable in the standard framework (see Hurwicz et. al., 1995).
} 
Each $i \in N$ is characterized by a consumption space $\mathbb{R}_{+}^{\ell}$ (where $x_{i}=$ $\left(x_{i 1}, \ldots, x_{i \ell}\right) \in \mathbb{R}_{+}^{\ell}$ is the $i$ 's commodity bundle), by an endowment vector $\omega_{i} \in \mathbb{R}_{+}^{\ell}$ and by a preference relation defined over $\mathbb{R}_{+}^{\ell}{ }^{6}{ }^{6}$ Let 0 denote the zero consumption bundle. Each $i$ 's preferences have a utility representation $u_{i}: \mathbb{R}_{+}^{\ell} \rightarrow \mathbb{R}$ which is continuous and quasi-concave on $\mathbb{R}_{+}^{\ell}$, and either strongly monotone on $\mathbb{R}_{+}^{\ell}$ or strongly monotone on $\mathbb{R}_{++}^{\ell}$ such that the utility of every interior consumption bundle is strictly higher than the utility of any consumption bundle on the boundary. $U$ is the class of all such utility functions, whereas $U_{i}$ is the class of admissible utility functions for $i$. The aggregate endowment is denoted by $\Omega \equiv \sum \omega_{i} \gg 0,{ }^{7}$ where the distribution of endowments $\left(\omega_{i}\right)_{i \in N} \in \mathbb{R}_{+}^{n \ell}$ is known and fixed.

Let $Q \equiv\left\{x_{i} \in \mathbb{R}_{+}^{\ell} \mid x_{i} \leq \Omega\right\}$. For each $i$, each $u_{i}$, and each $x_{i} \in Q$, $L\left(x_{i}, u_{i}\right) \equiv\left\{x_{i}^{\prime} \in Q \mid u_{i}\left(x_{i}\right) \geq u_{i}\left(x_{i}^{\prime}\right)\right\}$ denotes the weak lower contour set of agent $i$ for $u_{i}$ at $x_{i}$. An economy is specified by a list $u=\left(u_{i}\right)_{i \in N} \in$ $U_{N} \equiv \times_{i \in N} U_{i}$. An allocation is a list of bundles $x=\left(x_{1}, \ldots, x_{n}\right) \in \mathbb{R}_{+}^{n \ell}$, whereas a feasible allocation is an allocation $x=\left(x_{1}, \ldots, x_{n}\right) \in \mathbb{R}_{+}^{n \ell}$ such that $\sum x_{i} \leq \Omega$. Let $\mathbf{0}$ denote the feasible allocation in which every agent gets the zero consumption bundle. The set of all feasible allocations is denoted by $A$. Since no ambiguity can occur, $u$ has to be understood as an element of $U_{N}$ and we shall omit to refer it to $U_{N}$. The set of (Pareto) efficient allocations for $u$, denoted by $P(u)$, is $P(u) \equiv\{x \in A \mid$ there is no $y \in A$, with $u_{i}\left(y_{i}\right)>u_{i}\left(x_{i}\right)$ for all $\left.i\right\}$.

A social choice function $(S C F)$ is a single-valued mapping $f: U_{N} \rightarrow A$ such that for each $u, F_{i}(u) \neq 0$ for all $i \in N$ and $f(u) \in P(u)$. A social choice correspondence $(S C C)$ is a multi-valued mapping $F: U_{N} \rightarrow A$ such that for each $u, F(u)$ is a non-empty subset of $P(u)$ and for each $x \in F(u)$, $x_{i} \neq 0$ for all $i \in N$. Let $\mathcal{F}$ be the class of all such $S C C$ s. For each $u$, each allocation in $F(u)$ is said to be an $F$-optimal allocation at $u$. The nonzero property of any agent's consumption bundle at any $F$-optimal allocation represents the minimal condition of participation constraint. Among many elements of $\mathcal{F}$, we shall be concerned with the following well-known $S C C$ :

Walrasian correspondence, $W$ : For each $u, W(u) \equiv\{x \in A$ |there is $p \in \mathbb{R}_{+}^{\ell}$ such that for each $i, p \cdot x_{i}=p \cdot \omega_{i}$ and for each $y_{i} \in \mathbb{R}_{+}^{\ell}$,

\footnotetext{
${ }^{6}$ Hereafter unless otherwise specified lower case letters such as $h, i, j$, and $k$ should be understood as agents in $N$.

${ }^{7}$ When its bounds are not explicitly indicated, a summation should be understood to cover all agents.
} 


$$
\left.u_{i}\left(y_{i}\right)>u_{i}\left(x_{i}\right) \Rightarrow p \cdot y_{i}>p \cdot \omega_{i}\right\} .
$$

A mechanism is a pair $\gamma \equiv(M, g)$, where $M \equiv M_{1} \times \ldots \times M_{n}$, with each $M_{i}$ being a (non-empty) set and $g: M \rightarrow \mathbb{R}^{n \ell}$. It consists of a message space $M$, where $M_{i}$ is the message space for $i$, and an outcome function $g$ such that $g(m)=\left(g_{i}(m)\right)_{i \in N} \in \mathbb{R}^{n \ell}$ for each $m \in M$. A generic message for $i$ is denoted by $m_{i} \in M_{i}$, while a message profile is by $m \equiv\left(m_{1}, \ldots, m_{n}\right) \in M$. For $m \in M$ and $j$, let $m_{-j} \equiv\left(m_{1}, \ldots, m_{j-1}, m_{j+1}, \ldots, m_{n}\right) \in M_{-j} \equiv \times_{i \in N \backslash\{j\}} M_{i}$. Given $m_{-j} \in M_{-j}$ and $m_{j} \in M_{j},\left(m_{j}, m_{-j}\right)$ is the message profile consisting of $m_{j}$ and $m_{-j}$. The attainable set of $i$ at $m_{-i}$, the set of bundles that $i$ can induce when the other agents select $m_{-i}$, is denoted by $g\left(M_{i}, m_{-i}\right)$.

\subsection{Implementation with partially-honest agents}

For any $(i, u) \in N \times U_{N}$, let $\succcurlyeq_{i}^{u}$ be $i$ 's weak order over $M$ under the economy $u$. The asymmetric part of $\succcurlyeq_{i}^{u}$ is denoted by $\succ_{i}^{u}$, while the symmetric part is denoted by $\sim_{i}^{u}$. For any $u \in U_{N}, \succcurlyeq^{u}$ is the profile of weak orders over $M$ under the economy $u$; in other words, $\succcurlyeq^{u} \equiv\left(\succcurlyeq_{i}^{u}\right)_{i \in N}$.

For each mechanism $\gamma$, each $i$, and each economy $u$, let $T_{i}^{\gamma}(u ; F)$ be a nonempty subset of $M_{i}$, named a partially-honest domain of $i$ at $u$, by means of which, the notion of partially-honest agents is introduced as follows:

Definition 1 Agent $h$ has intrinsic preferences for honesty if for each mechanism $\gamma$ and each economy $u$, and for each $m_{-h}$, her weak order $\succcurlyeq_{h}^{u}$ over $M$ satisfies the following two properties.

(i) If $m_{h} \in T_{h}^{\gamma}(u ; F), m_{h}^{\prime} \notin T_{h}^{\gamma}(u ; F)$, and $u_{h}\left(g_{h}\left(m_{h}, m_{-h}\right)\right) \geq u_{h}\left(g_{h}\left(m_{h}^{\prime}, m_{-h}\right)\right)$, then $\left(\left(m_{h}, m_{-h}\right),\left(m_{h}^{\prime}, m_{-h}\right)\right) \in \succ_{h}^{u}$;

(ii) Otherwise, $\left(\left(m_{h}, m_{-h}\right),\left(m_{h}^{\prime}, m_{-h}\right)\right) \in \succcurlyeq_{h}^{u}$ if and only if $u_{h}\left(g_{h}\left(m_{h}, m_{-h}\right)\right) \geq$ $u_{h}\left(g_{h}\left(m_{h}^{\prime}, m_{-h}\right)\right)$.

An agent $h$ who has intrisic preferences for honesty is said to be a partiallyhonest agent.

The subset $T_{i}^{\gamma}(u ; F)$ specifies the domain of the messages given in the mechanism $\gamma$, on which $i$ places an intrinsic value for honest behavior. The notion of partially-honest domains is not only to specify at least a subset of 'truthful messages' given in the mechanism, but also to represent the extent of preferences for honesty each agent has in society. Therefore, though each element in a partially-honest domain is interpreted as a truthful message 
in the mechanism, some of 'truthful messages' would not belong to that domain if the intrinsic value for honesty is not placed on this message by the partially-honest agents. ${ }^{8}$

Definition 2 Agent $i$ has no intrinsic preference for honesty if for each mechanism $\gamma$ and each economy $u$, her weak order $\succcurlyeq_{i}^{u}$ over $M$ satisfies the following property: for each $m$ and $m^{\prime}$,

$$
\left(m, m^{\prime}\right) \in \succcurlyeq_{i}^{u} \text { if and only if } u_{i}\left(g_{i}(m)\right) \geq u_{i}\left(g_{i}\left(m^{\prime}\right)\right) \text {. }
$$

Unless stated otherwise, the following informational assumption holds throughout the paper.

Assumption 1 There are partially-honest agents in $N$, which is acknowledged by the mechanism designer, though she does not know their identities or their exact number.

The mechanism designer cannot exclude any agent from being partiallyhonest on the basis of information given by Assumption 1. To formalize this fact, let $\mathcal{H} \subseteq 2^{N} \backslash\{\varnothing\}$ be a non-empty class of non-empty subsets of $N$. Given the truly limited information injected by Assumption 1, in what follows we shall view $\mathcal{H}$ as the class of conceivable sets of partially-honest agents. Since no ambiguity can occur, $H$ has to be understood as an element of $\mathcal{H}$ and we shall omit to refer it to $\mathcal{H}$.

A mechanism $\gamma$ induces a class of (non-cooperative) games with partiallyhonest agents $\left\{\left(\gamma, \succcurlyeq^{u, H}\right) \mid(u, H) \in U_{N} \times \mathcal{H}\right\}$, where $\succcurlyeq^{u, H}$ denotes the profile of agents' weak orders over $M$ when $u$ is the underlying economy and $H$ is the set of partially-honest agents. Given a game $\left(\gamma, \succcurlyeq^{u, H}\right)$, we say that $m^{*}$ is a (pure strategy) Nash equilibrium with partially-honest agents at $u$ and $H$ if and only if for all $i,\left(m^{*},\left(m_{i}, m_{-i}^{*}\right)\right) \in \succcurlyeq_{i}^{u, H}$ for all $m_{i}$. Given a game $\left(\gamma, \succcurlyeq^{u, H}\right), N E\left(\gamma, \succcurlyeq^{u, H}\right)$ denotes the set of (Nash) equilibrium message profiles of $\left(\gamma, \succcurlyeq^{u, H}\right)$, whereas $N A\left(\gamma, \succcurlyeq^{u, H}\right)$ represents the corresponding set of (Nash) equilibrium allocations.

Definition 3 A mechanism $\gamma$ partially-honestly implements $F$ in Nash equilibria, or simply partially-honestly implements $F$ if and only if

$$
\text { for each } u \text { and each } H: F(u)=N A\left(\gamma, \succcurlyeq^{u, H}\right) \text {. }
$$

If such a mechanism exists, $F$ is partially-honestly implementable.

\footnotetext{
${ }^{8}$ It is consistent with the motivation of Dutta and Sen (2012), who discuss implementation problems with small preferences for honesty.
} 
Definition 3 is similar, but not identical to, the standard definition of implementation. ${ }^{9}$ First, the equilibrium allocations are given by the game $\left(\gamma, \succcurlyeq^{u, H}\right)$ rather than by the game $(\gamma, u)$. Second, the equivalence of the set of $F$-optimal allocations with the set of Nash equilibrium allocations is required not only for each economy $u$ but also for each conceivable set $H$.

\subsection{Natural price-quantity mechanisms with free-disposal}

Following Saijo et al. (1996, 1999), we now formalize the notion of implementation with partially-honest agents by natural price-quantity mechanisms with free-disposal. For each pair $\left(u_{i}, x_{i}\right) \in U_{i} \times Q, V_{i}\left(x_{i}, u_{i}\right) \equiv\left\{y_{i} \in\right.$ $\left.Q \mid u_{i}\left(x_{i}\right) \leq u_{i}\left(y_{i}\right)\right\}$ denotes the weak upper contour set of agent $i$ for $u_{i}$ at $x_{i}$. Given $\left(u_{i}, x_{i}\right)$, a price vector $p$ belonging to the unit simplex $\Delta$, that is, $p \in \Delta$, is said to be a sub-gradient of $u_{i}$ at $x_{i}$, denoted by $\nabla u_{i}\left(x_{i}\right)$, if $p \cdot x_{i}^{\prime} \geq p \cdot x_{i}$ for all $x_{i}^{\prime} \in V_{i}\left(x_{i}, u_{i}\right)$. The set of sub-gradients of $u_{i}$ at $x_{i}$, denoted by $\bar{\nabla} u_{i}\left(x_{i}\right)$, is called the sub-differential of $u_{i}$ at $x_{i}$.

For each $(x, u) \in Q^{n} \times U_{N}$, define the set of efficiency prices for $u$ at $x$ as

$$
\Pi(x, u) \equiv \bigcap_{i} \bar{\nabla} u_{i}\left(x_{i}\right) .
$$

Notice that $x \in P(u)$ if $\Pi(x, u)$ is non-empty. In words, $\Pi(x, u)$ consists of prices $p$ each of which is normal to a hyperplane separating the weak upper contour sets of all agents with $u$ at $x$.

For each economy $u$, each $F$-optimal allocation $x$ associated with $u$, and each efficiency price $p$ for $u$ at $x$, let us define the following sets.

$$
\begin{gathered}
F^{-1}(x, p) \equiv\left\{u^{\prime} \mid x \in F\left(u^{\prime}\right) \text { and } p \in \Pi\left(x, u^{\prime}\right)\right\}, \\
F_{!}^{-1}(x, p) \equiv\left\{u^{\prime} \mid x \in F\left(u^{\prime}\right) \text { and }\{p\}=\Pi\left(x, u^{\prime}\right)\right\}, \\
\Pi^{F}(x, u) \equiv\left\{p \in \Pi(x, u) \mid F_{!}^{-1}(x, p) \text { is non-empty }\right\},
\end{gathered}
$$

and

$$
\pi^{F}(x, u) \equiv \begin{cases}\Pi^{F}(x, u) & \text { if } \Pi^{F}(x, u) \text { is non-empty } \\ \Pi(x, u) & \text { otherwise. }\end{cases}
$$

\footnotetext{
${ }^{9}$ The two definitions are however identical if the family $\mathcal{H}$ is empty.
} 
Any $p \in \pi^{F}(x, u)$ is referred to as an efficiency price for $u$ and $F$ at $x .^{10}$ The reader should consult Saijo et al. $(1996,1999)$ for a discussion of $F^{-1}(x, p)$.

The difference between $\pi^{F}(x, u)$ and $\Pi(x, u)$ centers on whether it is possible to select efficiency prices from the set $\Pi(x, u)$ which are relevant for the $F$-optimal allocation $x$ at $u$. This point is particularly important where $F$ is the Walrasian correspondence (on this point see Saijo et al. 1999).

Definition 4 An $S C C F \in \mathcal{F}$ is partially-honestly implementable by a natural price-quantity mechanism if there exists a mechanism $\gamma=(M, g)$ such that:

(i) $\gamma$ partially-honestly implements $F$.

(ii) For each $i, M_{i}=\Delta \times Q$.

(iii) For each $u$, each $x \in F(u)$, and each $p \in \pi^{F}(x, u)$, if $m_{i}=\left(p, x_{i}\right)$ for each $i$, then $m \in N E\left(\gamma, \succcurlyeq^{u, H}\right)$ for each $H$ and $g(m)=x$.

(iv) $\gamma$ is feasible: $g(m) \in A$ for each $m \in M$.

(v) $\gamma$ satisfies the best response property: for all $i$, all $u_{i}$, and all $m_{-i}$, there exists $m_{i}$ such that $u_{i}\left(g_{i}\left(m_{i}, m_{-i}\right)\right) \geq u_{i}\left(g_{i}\left(m_{i}^{\prime}, m_{-i}\right)\right)$ for all $m_{i}^{\prime}$.

Call $\gamma$ a natural price-quantity mechanism (natural $p-q$ mechanism) if $\gamma$ satisfies the above requirements (ii)-(v).

The property (iii) is the forthrightness condition for a $p-q$ mechanism, which requires that in equilibrium each agent receives what she has announced as a bundle. ${ }^{11}$ Observe that our forthrightness condition differs from that of Saijo et al. (1999) because it applies only to efficiency prices in $\pi^{F}(x, u)$ rather than to those in $\Pi(x, u)$. We shall make use of this difference in the next sections, which will be relevant for the case where $F$ is the Walrasian correspondence.

\footnotetext{
${ }^{10}$ Note that, for any $p \in \Pi(x, u)$ with $x \in F(u)$, there always exists $u^{\prime} \in U_{N}$ such that $\{p\}=\Pi\left(x, u^{\prime}\right)$ whenever $U_{N}$ contains the set of all linear utility functions. Indeed, if $u_{i}^{\prime}\left(x_{i}\right)=p \cdot x_{i}$ for all $i \in N$, then $\{p\}=\Pi\left(x, u^{\prime}\right)$. However, note that $F_{!}^{-1}(x, p) \neq \varnothing$ does not necessarily follow from the case $\{p\}=\Pi\left(x, u^{\prime}\right)$. For instance, if $F \equiv W_{c}$ is the constrained Walrasian correspondence (on the definition of this, see Saijo et al. 1999), it is possible that $x \in W_{c}(u), p \in \Pi(x, u),\{p\}=\Pi\left(x, u^{\prime}\right)$, but $x \notin W_{c}\left(u^{\prime}\right)$, whenever $p$ is not a (constrained) Walrasian equilibrium price vector corresponding to $x \in W_{c}(u)$.

${ }^{11}$ The reader should refer to Dutta et al. (1995), Saijo et al. (1996), and Lombardi and Yoshihara (2013a) for a more discussion on forthrightness.
} 


\section{A contextualizing example and further con- nections to the literature}

The main works on implementation with partially-honest agents in economic environments are Dutta and Sen (2012) and Kartik et al. (2014). They assume that all agents have small intrinsic preferences for honesty, and by focussing on $S C F \mathrm{~s}$, have the merit of devising mechanisms that do not use any sort of "integer games" or "modulo games". Dutta and Sen (2012; Definition 11) provide a separability condition under which any $S C F$ is implementable in strictly dominant strategies. Kartik et al. (2014) offer a condition called separable punishment (Definition 2, p. 287) under which any SCF is implementable by a simple mechanism in two rounds of iterated deletion of strictly dominated strategies. This section is to show that their results do not extend to our problems provided that the attention is paid to $S C C$ s.

In Dutta and Sen (2012), an agent $i$ has intrinsic preferences for reporting honestly the true economy $u$, whereas in problems of implementation by $p$ $q$ mechanisms, at least two types of intrinsic preferences for honesty could be considered, as discussed in section 1 . The first one is that an agent $i$ has intrinsic preferences for truthfully reporting her own demand quantity $x_{i}$ that is also consistent with $F(u)$, which is formalized as follows.

Definition 5 Given a natural $p$ - $q$ mechanism $\gamma$, an economy $u$, and an $S C C$ $F$, a partially-honest agent $h$ has weak intrinsic preferences for honesty if her partially-honest domain is

$$
T_{h}^{\gamma}(u ; F) \equiv\left\{\left(p, x_{h}\right) \in \Delta \times Q \mid \exists x_{-h}:\left(x_{h}, x_{-h}\right) \in F(u)\right\} .
$$

A consumption bundle $x_{h}$ is said to be weakly truthful for $u$ if and only if $\left(x_{h}, x_{-h}\right) \in F(u)$ for some $x_{-h}$.

Lombardi and Yoshihara (2013b) propose a condition, called Monotonicity with Weak Honesty (M-WH), which is necessary for implementation by a natural $p-q$ mechanism provided that agents have weak intrinsic preferences for honesty. To define this condition, some notations should be introduced. Let $p \in \Delta$ and $x=\left(x_{i}\right)_{i \in N} \in Q^{n}$ be given. If $x \in F(u)$ and $p \in \pi^{F}(x, u)$ for some $u$, let

$$
\bar{F}^{-1}(x, p) \equiv\left\{u^{\prime} \mid x \in F\left(u^{\prime}\right) \text { and } p \in \pi^{F}\left(x, u^{\prime}\right)\right\}
$$


and for each $i$, let

$$
\Lambda_{i}^{F}(x, p) \equiv \bigcap_{u^{\prime} \in \bar{F}^{-1}(x, p)} L\left(x_{i}, u_{i}^{\prime}\right)
$$

Then:

Condition M-WH Let $H$ be given. For each $u$ and $u^{*}$, each $x \in F(u)$, and each $p \in \pi^{F}(x, u)$, if $\Lambda_{i}^{F}(x, p) \subseteq L\left(x_{i}, u_{i}^{*}\right)$ for each $i$ and $x \notin F\left(u^{*}\right)$, then for some $i \in H, x_{i} \neq x_{i}^{*}$ for each $x^{*} \in F\left(u^{*}\right)$.

M-WH is an intuitive condition: If the economy $u$ moves to a new economy $u^{*}$ in such a way that for each $i$ the weak lower contour set for $u_{i}^{*}$ at $x_{i}$ contains the set $\Lambda_{i}^{F}(x, p)$ and if $x$ is an $F$-optimal allocation at $u$ but is not at $u^{*}$, then there must exist a partially-honest agent $i$ for whom the bundle $x_{i}$ is not weakly truthful for $u^{*}$.

The necessity of $\mathbf{M - W H}$ for the implementation is also easy to confirm: Suppose that a natural $p-q$ mechanism $\gamma$ implements the efficient allocation rule $F$, and let us consider any set $H$ of partially-honest agents with weak intrinsic preferences for honesty and any two economies $u$ and $u^{*}$ meeting the premises of condtion M-WH. By the requirement (iii) of Definition 4 we are sure that the message profile $m$ where each agent $i$ announces $m_{i}=\left(p, x_{i}\right)$ is an equilibrium one of the game $\left(\gamma, \succcurlyeq^{u, H}\right)$ and that the outcome of the mechanism is the allocation $x=g(m)$. One way now to see the necessity of $\mathbf{M - W H}$ is to assume, contrary to the statement, that for each partiallyhonest agent $i \in H$ there exists an $F$-optimal allocation $x^{*}$ at $u^{*}$ such that $x_{i}^{*}=x_{i}$. Thus, for each agent in $H,\left(p, x_{i}\right)$ is weakly truthful for $u^{*}$ as well. Since no agent can find a profitable deviation from $m$, one can see that $x$ is an equilibrium outcome of the game $\left(\gamma, \succcurlyeq^{u^{*}, H}\right)$, which contradicts the supposition that $x$ is not an $F$-optimal allocation at $u^{*}$.

Theorem 1 in Lombardi and Yoshihara (2013b) implies that condition M-WH is also sufficient for implementation if free-disposal is allowed. From this, the following positive result for $S C F$ s can be seen readily:

Corollary 1 Assume that all agents have weak intrinsic preferences for honesty. Every efficient SCF is partially-honestly implementable by a natural $p-q$ mechanism. ${ }^{12}$

\footnotetext{
${ }^{12}$ The proof can easily be obtained from the proof of Theorem 1 in Lombardi and Yoshihara (2013b) by adapting it to economies with free-disposal. At the same time, the natural price-quantity mechanism can be constructed without using any form of "tail-chasing" construction, as in the proof of Theorem 1 of this paper, presented in the appendix.
} 
Note that this general positive result, although similar in flavor to (but not an extension of,) those established by both Dutta and Sen (2012) and Kartik et al. (2014), is obtained by a natural $p-q$ mechanism.

However, many interesting allocation rules are not single-valued, and if multi-valued allocation rules are concerned, there are still many interesting rules which are non-implementable - the Walrasian correspondence, $W$, is one of such examples. Example 1 below illustrates a violation of $\mathbf{M}-\mathbf{W H}$ for $W$ where all agents have weak intrinsic preferences for honesty.

Example 1 Suppose that all agents have weak (but not strong) intrinsic preferences for honesty. For the sake of simplicity, let $n=\ell=3$, and let $\omega_{i}=(1,1,1)$ for each $i$. Choose $u$ such that a Walrasian equilibrium allocation at $u$ is $x_{1}=(3,0,1), x_{2}=(0,3,0)$, and $x_{3}=(0,0,2)$, with the equilibrium price $p=\left(\frac{1}{6}, \frac{1}{3}, \frac{1}{2}\right)$. Let $x^{*}=\left(x_{1}^{*}, x_{2}^{*}, x_{3}^{*}\right), x^{* *}=$ $\left(x_{1}^{* *}, x_{2}^{* *}, x_{3}^{* *}\right)$, and $x^{* * *}=\left(x_{1}^{* * *}, x_{2}^{* * *}, x_{3}^{* * *}\right)$ be allocations in $A$ given by

$$
\begin{aligned}
\left(x_{1}^{*}, x_{2}^{*}, x_{3}^{*}\right) & =\left(x_{1},(0,2,0),(0,1,2)\right), \\
\left(x_{1}^{* *}, x_{2}^{* *}, x_{3}^{* *}\right) & =\left(\left(3,0, \frac{1}{4}\right), x_{2},\left(0,0, \frac{11}{4}\right)\right), \\
\left(x_{1}^{* * *}, x_{2}^{* * *}, x_{3}^{* * *}\right) & =\left(\left(3,0, \frac{1}{2}\right),\left(0,3, \frac{1}{2}\right), x_{3}\right) .
\end{aligned}
$$

Furthermore, as shown in the following Figures, for each $i$ there exists a continuous, quasi-concave and strongly monotone utility function $u_{i}^{*}$ such that the sub-gradient vector of $u_{i}^{*}$ at $x_{i}$ is

$$
\nabla u_{1}^{*}\left(x_{1}\right)=\left(\frac{1}{4}, \frac{1}{2}, \frac{1}{4}\right), \quad \nabla u_{2}^{*}\left(x_{2}\right)=\left(\frac{10}{33}, \frac{11}{33}, \frac{12}{33}\right), \text { and } \nabla u_{3}^{*}\left(x_{3}\right)=\left(\frac{1}{4}, \frac{1}{4}, \frac{1}{2}\right) \text {, }
$$

while the sub-gradient vector of $u_{i}^{*}$ at $x_{i}^{*}$, at $x_{i}^{* *}$, and at $x_{i}^{* * *}$ is, respectively,

$$
\begin{array}{llll}
\nabla u_{1}^{*}\left(x_{1}^{*}\right)=\nabla u_{1}^{*}\left(x_{1}\right), & \nabla u_{2}^{*}\left(x_{2}^{*}\right)=\left(\frac{1}{4}, \frac{1}{2}, \frac{1}{4}\right), & \text { and } \nabla u_{3}^{*}\left(x_{3}^{*}\right)=\left(\frac{1}{4}, \frac{1}{2}, \frac{1}{4}\right), \\
\nabla u_{1}^{*}\left(x_{1}^{* *}\right)=\left(\frac{10}{33}, \frac{11}{33}, \frac{12}{33}\right), & \nabla u_{2}^{*}\left(x_{2}^{* *}\right)=\nabla u_{2}^{*}\left(x_{2}\right), & \text { and } \nabla u_{3}^{*}\left(x_{3}^{* *}\right)=\left(\frac{10}{33}, \frac{11}{33}, \frac{12}{33}\right), \\
\nabla u_{1}^{*}\left(x_{1}^{* * *}\right)=\left(\frac{1}{4}, \frac{1}{4}, \frac{1}{2}\right), & \nabla u_{2}^{*}\left(x_{2}^{* * *}\right)=\left(\frac{1}{4}, \frac{1}{4}, \frac{1}{2}\right), & \text { and } \nabla u_{3}^{*}\left(x_{3}^{* * *}\right)=\nabla u_{3}^{*}\left(x_{3}\right) .
\end{array}
$$

By construction, $\Lambda_{i}^{W}(x, p) \subseteq L\left(x_{i}, u_{i}^{*}\right)$ for all $i$ and $x \notin W\left(u^{*}\right)$ because $x_{1} \notin \arg \max _{p \cdot x_{1}^{\prime} \leq p \cdot \omega_{1}} u_{1}^{*}\left(x_{1}^{\prime}\right)$. On the other hand, $x^{*} \in W\left(u^{*}\right)$ with the equilibrium price $p^{*}=\left(\frac{1}{4}, \frac{1}{2}, \frac{1}{4}\right), x^{* *} \in W\left(u^{*}\right)$ with the equilibrium price $p^{* *}=\left(\frac{10}{33}, \frac{11}{33}, \frac{12}{33}\right)$, and $x^{* * *} \in W\left(u^{*}\right)$ with the equilibrium price $p^{* * *}=\left(\frac{1}{4}, \frac{1}{4}, \frac{1}{2}\right)$. We established that $x^{*} \in W\left(u^{*}\right)$ and $x_{1}^{*}=x_{1}, x^{* *} \in$ $W\left(u^{*}\right)$ and $x_{2}^{* *}=x_{2}$, and $x^{* * *} \in W\left(u^{*}\right)$ and $x_{3}^{* * *}=x_{3}$, in violation of condition M-WH. 

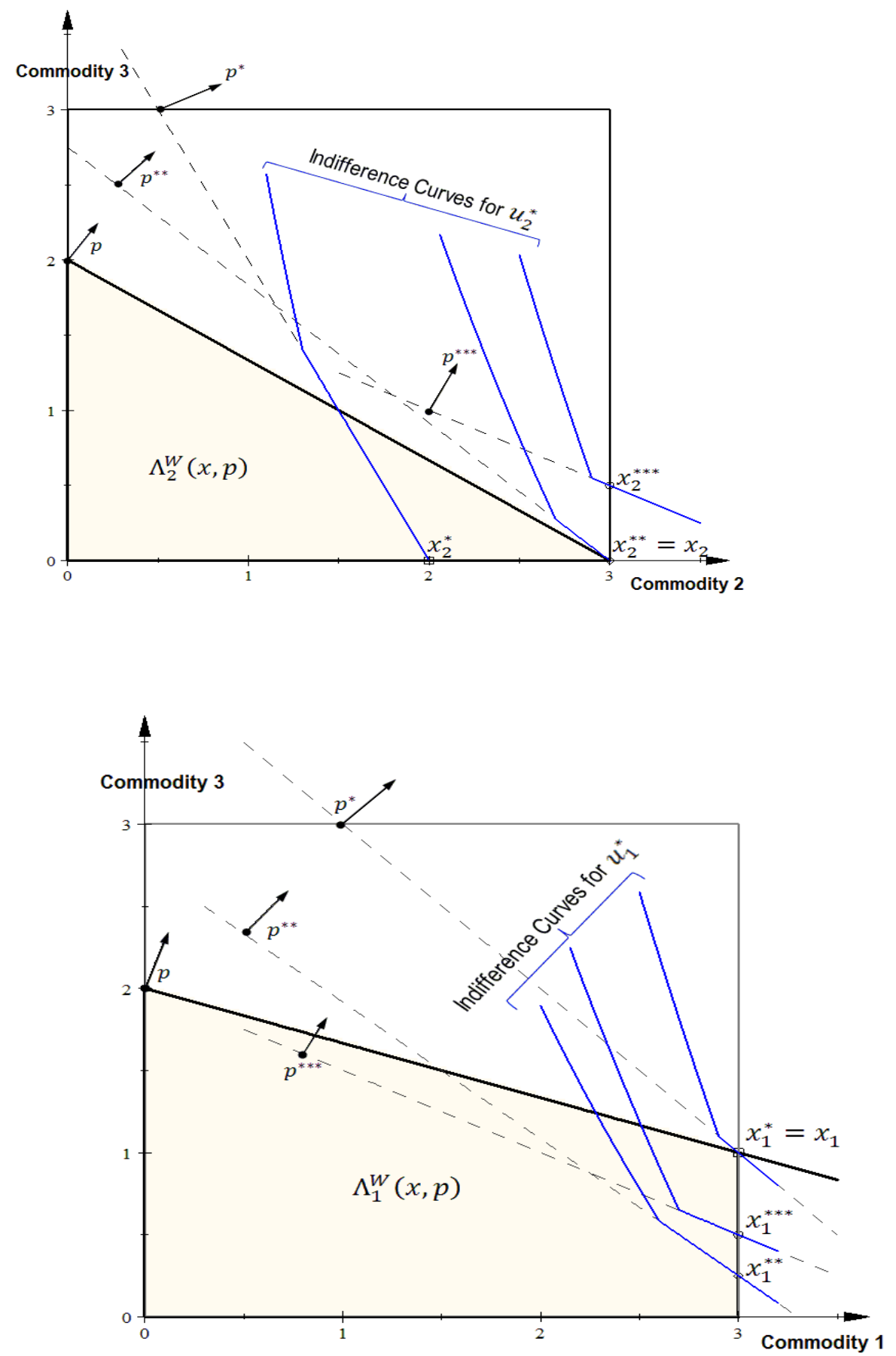


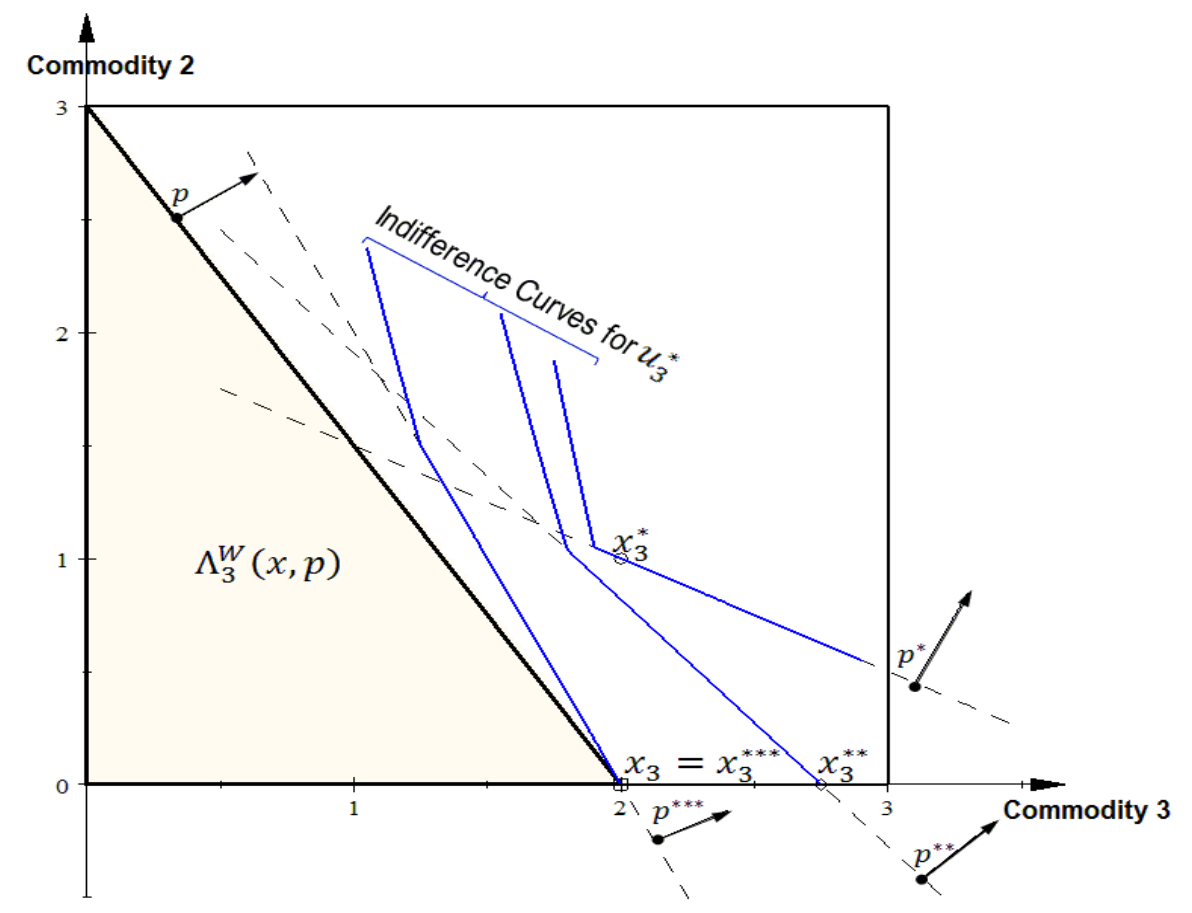

The main source of discrepancy between Example 1 and the existing results in separable environments is that $W$ is not a single-valued allocation rule. Therefore, if we want to discuss the implementability of $W$ by using the existing results, this would force us to perform selections from $W$. This may not be wise because a multi-valued allocation rule satisfying a property of interest need not admit any single-valued selection with that property. ${ }^{13}$

Example 1 suggests that weak intrinsic preferences for honesty are too weak to ensure the implementability of $W$. Indeed, under any mechanism $\gamma$ satisfying Definition 4(ii)-(v), if each agent in Example 1 announces $m_{i}=$ $\left(p, x_{i}\right)$ at the economy $u^{*}$, then the outcome should be $g(m)=x$ by Definition 4(iii). Then, since $p$ cannot be a Walrasian equilibrium price vector at $u^{*}$, no agent's announcement is a truthful point in the graph of her demand correspondence. However, $\left(p, x_{i}\right) \in T_{i}^{\gamma}\left(u^{*} ; W\right)$ holds for any agent because she only concerns about the truthfulness of her demand quantity announcement, thus $x \in W(u) \backslash W\left(u^{*}\right)$ could be a Nash equilibrium allocation at $u^{*}$.

\footnotetext{
${ }^{13}$ As an example, we may think of the upper-hemicontinuity of $W$ on exchange economies with linear preferences.
} 


\section{The main result}

In this section, we introduce the second type of preferences for honesty, called strong intrinsic preferences for honesty. Then, we show that whenever there exists at least one agent having strong intrinsic preferences for honesty, an $S C C$ is implementable by a natural $p$ - $q$ mechanism if and only if it satisfies a condition called Monotonicity/Punishment with Strong Honesty (MP-SH). Our constructive proof does not rely on any sort of "tail-chasing" construction that is common in the constructive proofs ${ }^{14}$ of the literature. An important implication of our result is that $W$ is implementable.

The strong intrinsic preference for honesty cares about honestly announcing a particular point in the graph of an agent's true demand correspondence, that is, a pair $\left(p, x_{i}\right)$ such that $x_{i}$ is consistent with $F(u)$, in that $\left(x_{i}, x_{-i}\right) \in F(u)$ for some $x_{-i}$ and $p$ is an efficiency price for $u$ and $F$ at $\left(x_{i}, x_{-i}\right)$. For instance, in the case of $W$, an agent $i$ satisfies her strong intrinsic preferences for honesty if she reports a true pair of a Walrasian equilibrium price vector and an associated equilibrium demand vector, rather than a disequilibrium pair of prices and demand quantity. ${ }^{15}$

Formally, this notion can be stated as follows.

Definition 6 Given a natural $p$ - $q$ mechanism $\gamma$, an economy $u$, and an $S C C$ $F$, a partially-honest agent $h$ has strong intrinsic preferences for honesty if her partially-honest domain is

$T_{h}^{\gamma}(u ; F) \equiv\left\{\left(p, x_{h}\right) \in \Delta \times Q \mid \exists x_{-h}:\left(x_{h}, x_{-h}\right) \in F(u)\right.$ and $\left.p \in \pi^{F}\left(\left(x_{h}, x_{-h}\right), u\right)\right\}$.

Definition 7 A pair $\left(p, x_{h}\right) \in \Delta \times Q$ is said to be strongly truthful for $u$ if and only if $\left(x_{h}, x_{-h}\right) \in F(u)$ and $p \in \pi^{F}\left(\left(x_{h}, x_{-h}\right), u\right)$ for some $x_{-h}$.

To facilitate our discussion, following Saijo et al. (1996, 1999), define the set of potential deviators as

$$
I^{F}(p, x) \equiv\left\{i \in N \mid \bar{F}^{-1}\left(\left(\Omega-\sum_{j \neq i} x_{j}, x_{-i}\right), p\right) \neq \varnothing\right\} .
$$

\footnotetext{
${ }^{14}$ See Maskin (1999). For the criticism against such construction, see Jackson (1992).

${ }^{15} \mathrm{An}$ analogous view can be applied to any $F$-optimal allocation and its associated efficiency price vector under certain conditions, because of the second welfare theorem.
} 
For each $i$, each $u_{i}$ and each $x_{i}$, let $\partial L\left(x_{i}, u_{i}\right)$ denote the upper boundary of $L\left(x_{i}, u_{i}\right)$, that is, $\partial L\left(x_{i}, u_{i}\right) \equiv\left\{x_{i}^{\prime} \in L\left(x_{i}, u_{i}\right) \mid u_{i}\left(x_{i}\right)=u_{i}\left(x_{i}^{\prime}\right)\right\}$.

We can now state our necessary and sufficient condition for implementation with partially-honest agents by natural $p$ - $q$ mechanisms under Definition 6.

Condition MP-SH Let $H, F$ and $(p, x) \in \Delta \times Q^{n}$ be given. Then:

(i) Let $I^{F}(p, x)=N$. Then, there exists $\mathbf{z}(p, x) \in A$, with $\mathbf{z}(p, x)=x$ if $\sum x_{i}=\Omega$, such that $\mathbf{z}_{i}(p, x) \in \Lambda_{i}^{F}\left(\left(\Omega-\sum_{j \neq i} x_{j}, x_{-i}\right), p\right)$ for each agent $i$.

(ii) Let $1 \leq \# I^{F}(p, x) \leq n-1$. Then, there exists $\overline{\mathbf{z}}(p, x) \in A$ such that for each $i, \overline{\mathbf{z}}_{i}(p, x) \in \Lambda_{i}^{F}\left(\left(\Omega-\sum_{j \neq i} x_{j}, x_{-i}\right), p\right)$ if $i \in I^{F}(p, x)$ and $\left(p, x_{i}\right)$ is strongly truthful for some $u$, or else $\overline{\mathbf{z}}_{i}(p, x)=0$.

(iii) For each agent $i$, there exists a map $z_{i}\left(\cdot ;\left(p, x_{-i}\right)\right): \Delta \times Q \rightarrow Q$ such that for each $\left(p^{\prime}, x_{i}^{\prime}\right)$ :

(iii.a) If $\left(p^{\prime}, x_{i}^{\prime}\right)$ is strongly truthful for some $u^{\prime}$ and $i \in I^{F}\left(p,\left(x_{i}^{\prime}, x_{-i}\right)\right)$,

$$
\begin{aligned}
& \text { then } z_{i}\left(\left(p^{\prime}, x_{i}^{\prime}\right) ;\left(p, x_{-i}\right)\right) \in \Lambda_{i}^{F}\left(\left(\Omega-\sum_{j \neq i} x_{j}, x_{-i}\right), p\right), \text { with } \\
& z_{i}\left(\left(p^{\prime}, x_{i}^{\prime}\right) ;\left(p, x_{-i}\right)\right)=\left\{\begin{array}{cc}
\mathbf{z}_{i}(p, x) & \text { if } p^{\prime}=p \text { and } I^{F}\left(p,\left(x_{i}^{\prime}, x_{-i}\right)\right)=N, \\
\overline{\mathbf{z}}_{i}\left(p,\left(x_{i}^{\prime}, x_{-i}\right)\right) & \text { if } p^{\prime}=p \text { and } 1 \leq \# I^{F}\left(p,\left(x_{i}^{\prime}, x_{-i}\right)\right) \leq n-1 .
\end{array}\right.
\end{aligned}
$$

(iii.b) Otherwise, $z_{i}\left(\left(p^{\prime}, x_{i}^{\prime}\right) ;\left(p, x_{-i}\right)\right)=0$.

(iv) For each $u$, if $I^{F}(p, x)=N, \Lambda_{k}^{F}\left(\left(\Omega-\sum_{j \neq k} x_{j}, x_{-k}\right), p\right) \subseteq L\left(\mathbf{z}_{k}(p, x), u_{k}\right)$

for each $k$, and $\mathbf{z}(p, x) \notin F(u)$, then there is an agent $i \in H$ for whom (a) $\left(p, x_{i}\right)$ is not strongly truthful for $u$; and (b) there exists a strongly truthful pair $\left(p^{\prime}, x_{i}^{\prime}\right)$ for $u$ such that $z_{i}\left(\left(p^{\prime}, x_{i}^{\prime}\right) ;\left(p, x_{-i}\right)\right) \in$ $\partial L\left(\mathbf{z}_{i}(p, x), u_{i}\right)$.

This condition applies to cases where all agents announce the same price vector $p$. Firstly, if the announced profile of quantities $x$ is $F$-optimal for some economy and $p$ is an efficiency price vector for this economy and $F$ at $x$, then MP-SH(i) sets $\mathbf{z}(p, x)=x$. Otherwise, it implies that there are potential deviators in coordinating announcement of quantities, and if all agents are 
potential deviators, MP-SH(i) selects a feasible allocation and denotes it by $\mathbf{z}(p, x)$; otherwise, MP-SH(ii) selects a feasible allocation and denotes it by $\overline{\mathbf{z}}(p, x)$. In either case, any potential deviator $i \in I^{F}(p, x)$ can be punished by a bundle within the intersection set $\Lambda_{i}^{F}\left(\left(\Omega-\sum_{j \neq i} x_{j}, x_{-i}\right), p\right)$, which is not better than $\Omega-\sum_{j \neq i} x_{j}$ whatever $i$ 's true utility function is.

Then, MP-SH(iii)-(iv) addresses the case where agents change their messages, and specifies a map $z_{i}\left(\cdot ;\left(p, x_{-i}\right)\right): \Delta \times Q \rightarrow Q$ which identifies how to transform agent $i$ 's bundle when she shifts her message from $\left(p, x_{i}\right)$ to $\left(p^{\prime}, x_{i}^{\prime}\right)$. MP-SH(iii) basically states that $z_{i}\left(\left(p, x_{i}\right) ;\left(p, x_{-i}\right)\right)=\mathbf{z}_{i}(p, x)$ or $\overline{\mathbf{z}}_{i}(p, x)$ whenever the profile $\left(\left(p, x_{i}\right),\left(p, x_{-i}\right)\right)$ corresponds to the premises of MP-SH(i)-(ii). The most important part is MP-SH(iv): It addresses the case where each agent $i$ 's lower contour set of an economy $u$ at $\mathbf{z}_{i}(p, x)$ contains the intersection set $\Lambda_{i}^{F}\left(\left(\Omega-\sum_{j \neq i} x_{j}, x_{-i}\right), p\right)$ but $\mathbf{z}(p, x)$ is not $F$-optimal at $u$. To ensure the implementability in terms of Definition 4 , $\mathbf{z}(p, x)$ should not be a Nash equilibrium allocation at $u$ under any natural $p-q$ mechanism. However, if the standard framework is presumed, this case implies that there can be a natural $p-q$ mechanism under which $\mathbf{z}(p, x)$ is a Nash equilibrium at $u$, as Saijo et. al $(1996,1999)$ suggest. However, under Assumption 1 with strong intrinsic preferences for honesty, to ensure the implementability of $F, \mathbf{M P}-\mathbf{S H}(\mathrm{iv})$ exploits the existence of at least one partially-honest agent $i \in H$ for each given $H \in \mathcal{H}$, whose strong intrinsic preferences for honesty are not satisfied in the current message profile $(p, x)$, and specifies the bundle $z_{i}\left(\left(p^{\prime}, x_{i}^{\prime}\right) ;\left(p, x_{-i}\right)\right)$ by which she can be better off when she shifts to a strongly truthful message $\left(p^{\prime}, x_{i}^{\prime}\right)$.

We shall now establish our main result.

Theorem 1 Let Assumption 1 hold in conjunction with strong intrinsic preferences for honesty. An $S C C F \in \mathcal{F}$ is partially-honestly implementable by a natural $p-q$ mechanism if and only if it satisfies $\boldsymbol{M P}-\boldsymbol{S H}$.

Let us turn now to a brief discussion of the implications of Theorem 1. While its main implication is a positive one, as given in Theorem 2 below, we note an impossibility result: the efficient egalitarian-equivalent correspondence does not satisfy MP-SH. ${ }^{16}$

\footnotetext{
${ }^{16}$ See Lombardi and Yoshihara (2013b).
} 
Theorem 2 Let Assumption 1 hold in conjunction with strong intrinsic preferences for honesty. Let $U_{N}=\underbrace{U \times \ldots \times U}_{n \text { times }}$. Then, $W$ is partially-honestly implementable by a natural $p-q$ mechanism.

Let us briefly present the key details that lie behind the proof of Theorem 2. Basically, we need to prove two things. Firstly, by specifying the requirements of conditions MP-SH(i)-(iii) for the Walrasian rule we need to prove the existence of consumption bundles satisfying those requirements. Secondly, we need to prove the assertion that the Walrasian rule satisfies condition MP-SH(iv).

Let us start with the first of these. To prove that the consumption bundles specified in conditions MP-SH(i)-(iii) belong to the set $\Lambda_{i}^{W}\left(\left(\Omega-\sum_{j \neq i} x_{j}, x_{-i}\right), p\right)$, we begin by setting the allocation $\mathbf{z}(p, x)$ of condition MP-SH(i) equal to $x$ if this allocation is such that the amount of each commodity is equal to the total amount available from the economy or else, equal to the zero consumption allocation. In addition, we set the allocation $\overline{\mathbf{z}}(p, x)$ of condition $\mathbf{M P}-\mathbf{S H}(\mathrm{ii})$ equal to the zero consumption allocation. Finally, we define agent $i$ 's function $z_{i}\left(\cdot ;\left(p, x_{-i}\right)\right)$ as follows. In all cases in which the requirements of condition MP-SH(i) are met, we set $z_{i}\left(\left(p^{\prime}, x_{i}^{\prime}\right) ;\left(p, x_{-i}\right)\right)$ equal to $\mathbf{z}_{i}(p, x)$. For all remaining cases, we set $z_{i}\left(\left(p^{\prime}, x_{i}^{\prime}\right) ;\left(p, x_{-i}\right)\right)$ equal to $\Omega-\sum_{j \neq i} x_{j}$ if the pair $\left(p^{\prime}, x_{i}^{\prime}\right)$ is strongly truthful for an economy and $p^{\prime} \neq p$ or else, equal to the zero consumption bundle. From this, proving the verification of conditions MP-SH(i)-(iii) should be as expected.

To prove that the Walrasian rule satisfies condition MP-SH(iv), a key step here is to show that for each economy $u$ and each Walrasian allocation $x$ associated with $u$, the set of efficiency prices for $u$ and $W$ at $x$ consists only of Walrasian equilibrium prices for $x$. In other words, to show that $p \cdot x_{i}=p \cdot \omega_{i}$ for each agent $i$ if the price vector $p$ belongs to $\pi^{W}(u, x)$. To prove this, we start by observing that, by our domain supposition, the set of efficiency prices for $u$ and $W$ defined in (3) is never empty and so $\pi^{W}(u, x)=\Pi^{W}(u, x)$. Then, for no economy can the pair $(x, p)$ constitute a Walrasian equilibrium if $p$ is an efficiency price for $u$ at $x$ such that $p \cdot x_{i} \neq p \cdot \omega_{i}$ for some agent $i$. This leads straightforwardly to the conclusion that the price vector $p$ cannot be an efficiency price for $u$ and $W$ at $x$ if $p$ is an efficiency price for $u$ at $x$ such that $p \cdot x_{i} \neq p \cdot \omega_{i}$ for some agent $i$. This step also provided us with the familiar expression for the set $\Lambda_{i}^{W}(x, p)$ : For each economy $u$ and each Walrasian allocation $x$ associated with $u$, the set $\Lambda_{i}^{W}(x, p)$ coincides with the 
constrained Walrasian budget set. ${ }^{17}$

Let us add a few additional observations about its completion. Suppose that the hypotheses of condition MP-SH(iv) hold. Firstly, this can only be the case if $\mathbf{z}(p, x)=x$. This provides us with the following observation: $(x, p)$ is a Walrasian equilibrium for an economy $u^{\prime}$ and $p$ is an efficiency price for $W$ and $u^{\prime}$ at $x$. Secondly, since $x$ does not belong to $W(u)$, it follows that at prices $p$ there is an agent $k$ for whom the utility-maximizing bundle is outside the set of feasible ones. This leads to this key observation: The price vector $p$ cannot be paired with any Walrasian equilibrium allocation for the economy $u$. With the preceding observations and the help of the above definition of $z_{i}\left(\cdot ;\left(p, x_{-i}\right)\right)$, the verification of condition MP-SH(iv) should not present any difficulties. Indeed, let us consider any agent $i$ in $H$ who has strong intrinsic preferences for honesty. From the preceding key observation it is clear the pair $\left(p, x_{i}\right)$ is not strongly truthful for $u$. This verifies part (a) of the conclusion of condition MP-SH(iv). Let us consider any pair $\left(p^{\prime}, x_{i}^{\prime}\right)$ which is instead strongly truthful for $u$. Since the price vector $p$ cannot be paired with any Walrasian equilibrium allocation for the economy $u$, it follows that $p^{\prime} \neq p$. Note also that since, by supposition, $I^{W}(p, x)=N$, it follows that agent $i$ belongs to $I^{W}\left(p,\left(x_{i}^{\prime}, x_{-i}\right)\right)$. Lastly, from the definition of $z_{i}\left(\left(p^{\prime}, x_{i}^{\prime}\right) ;\left(p, x_{-i}\right)\right)$ one has that $z_{i}\left(\left(p^{\prime}, x_{i}^{\prime}\right) ;\left(p, x_{-i}\right)\right)=\Omega-\sum_{j \neq i} x_{j}=x_{i}$. From this, one can easily see that the Walrasian rule satisfies also part (b) of the conclusion of condition MP-SH(iv).

\section{Concluding remarks}

In this paper, weak and strong intrinsic preferences for honesty are introduced in allocation problems of pure exchange economies with free-disposal, and implementation problems by natural $p-q$ mechanisms where agents have weak/strong intrinsic preferences for honesty are studied. First, the Walrasian rule is not implementable where all agents have weak (but not strong) intrinsic preferences for honesty. Second, we offer a condition called Monotonicity/Punishment with Strong Honesty and show that not only does this condition fully identify the class of efficient rules that are implementable when at least one agent has strong intrinsic preferences for honesty, but that the Walrasian rule also satisfies it. Thirdly, we observed that the efficient egalitarian-

\footnotetext{
${ }^{17}$ The constrained Walrasian budget set is the intersection of the standard budget set (defined by $p$ and $\omega_{i}$ ) with $Q$.
} 
equivalent correspondence is not partially-honestly implementable by natural $p-q$ mechanisms.

Similar to the results of Dutta and Sen (2012) and Kartik et al. (2014), free-disposal plays a crucial role for our results as well. When feasibility constraints are imposed with an equality, the analysis of implementation problems by natural $p$ - $q$ mechanisms is more difficult, as it requires the construction of an outcome function satisfying the additional property of balance: The sum of consumption bundles assigned to agents must always be equal to the aggregate endowment. This analysis is undertaken in Lombardi and Yoshihara (2013b).

\section{Appendix}

The following proofs were relegated from the text.

Proof of Theorem 1. Suppose that an $S C C F \in \mathcal{F}$ is partially-honestly implementable by a natural $p-q$ mechanism $\gamma=(M, g)$. We show that $F$ satisfies MP-SH. Let $(p, x) \in \Delta \times Q^{n}$ and $H$ be given. Let $\bar{m}_{k}=\left(p, x_{k}\right) \in$ $\Delta \times Q$ for each $k$ and $\bar{m} \equiv\left(\bar{m}_{k}\right)_{k \in N}$.

Let $I^{F}(p, x)=N$. Then for each $i, \bar{F}^{-1}\left(\left(x_{-i}, \Omega-\sum_{k \neq i} x_{k}\right), p\right) \neq$ $\varnothing$. Take any $i$ and any $u^{\prime} \in \bar{F}^{-1}\left(\left(x_{-i}, \Omega-\sum_{k \neq i} x_{k}\right), p\right)$. Moreover, let $m=\left(\bar{m}_{-i},\left(p, \Omega-\sum_{k \neq i} x_{k}\right)\right)$. Since $\left(x_{-i}, \Omega-\sum_{k \neq i} x_{k}\right) \in F\left(u^{\prime}\right)$ and $p \in$ $\pi^{F}\left(\left(x_{-i}, \Omega-\sum_{k \neq i} x_{k}\right), u^{\prime}\right)$, Definition 4(iii) implies $g(m)=\left(x_{-i}, \Omega-\sum_{k \neq i} x_{k}\right)$ and $m \in N E\left(\gamma, \succcurlyeq^{u^{\prime}, H^{\prime}}\right)$ for each $H^{\prime}$, so that $g\left(M_{i}, \bar{m}_{-i}\right) \subseteq L\left(\Omega-\sum_{k \neq i} x_{k}, u_{i}^{\prime}\right)$. Since $u^{\prime}$ is arbitrary, $g_{i}\left(M_{i}, \bar{m}_{-i}\right) \subseteq \Lambda_{i}^{F}\left(\left(x_{-i}, \Omega-\sum_{k \neq i} x_{k}\right), p\right)$. Since $i$ is arbitrary, $g_{i}\left(M_{i}, \bar{m}_{-i}\right) \subseteq \Lambda_{i}^{F}\left(\left(x_{-i}, \Omega-\sum_{k \neq i} x_{k}\right), p\right)$ for each $i$. Let $\mathbf{z}(p, x)=g(\bar{m})$. Then, for each $i, \mathbf{z}_{i}(p, x) \in \Lambda_{i}^{F}\left(\left(x_{-i}, \Omega-\sum_{k \neq i} x_{k}\right), p\right)$. If $\sum x_{i}=\Omega$, then $\Omega-\sum_{k \neq i} x_{k}=x_{i}$ for each $i$, so that by Definition 4(iii), $g_{i}(\bar{m})=x_{i}$ for each $i$. Thus, $F$ satisfies MP-SH(i).

Suppose that $1 \leq \# I^{F}(p, x) \leq n-1$. By the same arguments as in the preceding paragraph, $g_{i}\left(M_{i}, \bar{m}_{-i}\right) \subseteq \Lambda_{i}^{F}\left(\left(x_{-i}, \Omega-\sum_{k \neq i} x_{k}\right), p\right)$ for all $i \in I^{F}(p, x)$. For each $i$ define $\overline{\mathbf{z}}_{i}(p, x)$ as follows: If $i \in I^{F}(p, x)$ and 
$\bar{m}_{i}=\left(p, x_{i}\right)$ is strongly truthful for some $u$, then $\overline{\mathbf{z}}_{i}(p, x)=g_{i}(\bar{m})$, or else $\overline{\mathbf{z}}_{i}(p, x)=0$. By this definition, $\overline{\mathbf{z}}_{i}(p, x) \in \Lambda_{i}^{F}\left(\left(x_{-i}, \Omega-\sum_{k \neq i} x_{k}\right), p\right)$ if $i \in I^{F}(p, x)$ and $\left(p, x_{i}\right)$ is strongly truthful for some $u$, and that $\overline{\mathbf{z}}(p, x) \in A$. Therefore, $F$ satisfies MP-SH(ii).

Fix an arbitrary $i$. Define the real-valued function $z_{i}\left(\cdot ;\left(p, x_{-i}\right)\right): \Delta \times Q \rightarrow$ $Q$ as follows: For each $\left(p^{\prime}, x_{i}^{\prime}\right)$,

1. If $\left(p^{\prime}, x_{i}^{\prime}\right)$ is strongly truthful for some $u^{\prime}, i \in I^{F}\left(p,\left(x_{i}^{\prime}, x_{-i}\right)\right)$, and $p^{\prime} \neq$ $p$, then $z_{i}\left(\left(p^{\prime}, x_{i}^{\prime}\right) ;\left(p, x_{-i}\right)\right)=g_{i}\left(\left(p^{\prime}, x_{i}^{\prime}\right), \bar{m}_{-i}\right)$.

2. If $\left(p^{\prime}, x_{i}^{\prime}\right)$ is strongly truthful for some $u^{\prime},\left(p^{\prime}, x_{i}^{\prime}\right)=\left(p, x_{i}\right)$, and $I^{F}(p, x)=$ $N$, then $z_{i}\left(\left(p, x_{i}\right) ;\left(p, x_{-i}\right)\right)=g_{i}\left(\left(p, x_{i}\right), \bar{m}_{-i}\right)$.

3. If $\left(p^{\prime}, x_{i}^{\prime}\right)$ is strongly truthful for some $u^{\prime}, i \in I^{F}\left(p,\left(x_{i}^{\prime}, x_{-i}\right)\right), p^{\prime}=$ $p$, and $1 \leq \# I^{F}\left(p,\left(x_{i}^{\prime}, x_{-i}\right)\right) \leq n-1$, then $z_{i}\left(\left(p, x_{i}^{\prime}\right) ;\left(p, x_{-i}\right)\right)=$ $g_{i}\left(\left(p, x_{i}^{\prime}\right), \bar{m}_{-i}\right)$.

4. Otherwise, $z_{i}\left(\left(p^{\prime}, x_{i}^{\prime}\right) ;\left(p, x_{-i}\right)\right)=0$.

By the definition, $z_{i}\left(\left(p, x_{i}\right) ;\left(p, x_{-i}\right)\right)=\mathbf{z}_{i}(p, x)$ for case 2 , while $z_{i}\left(\left(p, x_{i}\right) ;\left(p, x_{-i}\right)\right)=$ $\overline{\mathbf{z}}_{i}(p, x)$ for case 3 . For case 1 , since $i \in I^{F}\left(p,\left(x_{i}^{\prime}, x_{-i}\right)\right), I^{F}\left(p,\left(\Omega-\sum_{k \neq i} x_{k}, x_{-i}\right)\right)=$ $N$. By the same arguments as in the proof of $\mathbf{M S - S H}(\mathrm{i}), g_{i}\left(M_{i}, \bar{m}_{-i}\right) \subseteq$ $\Lambda_{i}^{F}\left(\left(x_{-i}, \Omega-\sum_{k \neq i} x_{k}\right), p\right)$. Therefore, by the definition, $z_{i}\left(\left(p^{\prime}, x_{i}^{\prime}\right) ;\left(p, x_{-i}\right)\right) \in$ $\Lambda_{i}^{F}\left(\left(x_{-i}, \Omega-\sum_{k \neq i} x_{k}\right), p\right)$ even for case 1 . Since $i$ is arbitrary, $F$ satisfies MP-SH(iii).

Fix any $u$. Let $I^{F}(p, x)=N, \Lambda_{k}^{F}\left(\left(x_{-k}, \Omega-\sum_{j \neq k} x_{j}\right), p\right) \subseteq L\left(\mathbf{z}_{k}(p, x), u_{k}\right)$ for each $k$, and $\mathbf{z}(p, x) \notin F(u)$. Since $g_{k}\left(M_{k}, \bar{m}_{-k}\right) \subseteq \Lambda_{k}^{F}\left(\left(x_{-k}, \Omega-\sum_{j \neq k} x_{j}\right), p\right)$ and $\mathbf{z}_{k}(p, x)=g_{k}(\bar{m})$ for each $k, g_{k}\left(M_{k}, \bar{m}_{-k}\right) \subseteq L\left(\mathbf{z}_{k}(p, x), u_{k}\right)$ holds for each $k$. Since $\mathbf{z}(p, x) \notin N A\left(\gamma, \succcurlyeq^{u, H}\right)$, it follows that for some $i \in H,\left(p, x_{i}\right)$ is not strongly truthful for $u$. Moreover, since $\mathbf{z}(p, x) \notin N A\left(\gamma, \succcurlyeq^{u, H}\right)$ and $g_{i}\left(M_{i}, \bar{m}_{-i}\right) \subseteq L\left(\mathbf{z}_{i}(p, x), u_{i}\right)$, for the agent $i \in H$ there exists a strongly truthful $\left(p^{\prime}, x_{i}^{\prime}\right)$ for $u$ such that $g_{i}\left(\left(p^{\prime}, x_{i}^{\prime}\right), \bar{m}_{-i}\right) \in \Lambda_{i}^{F}\left(\left(x_{-i}, \Omega-\sum_{j \neq i} x_{j}\right), p\right)$ and $g_{i}\left(\left(p^{\prime}, x_{i}^{\prime}\right), \bar{m}_{-i}\right) \in \partial L\left(\mathbf{z}_{i}(p, x), u_{i}\right)$. By $i \in I^{F}\left(p,\left(x_{i}^{\prime}, x_{-i}\right)\right)$ and the definition of $z_{i}\left(\cdot ;\left(p, x_{-i}\right)\right), z_{i}\left(\left(p^{\prime}, x_{i}^{\prime}\right) ;\left(p, x_{-i}\right)\right)=g_{i}\left(\left(p^{\prime}, x_{i}^{\prime}\right), \bar{m}_{-i}\right)$. Thus, $F$ satisfies MP-SH(iv). Hence, $F$ satisfies MP-SH. 
Conversely, suppose that $F \in \mathcal{F}$ satisfies MP-SH. Denote the boundary set of $\Delta$ by $\partial \Delta$. For each $(x, p) \in Q^{n} \times \Delta, \partial \Lambda_{i}^{F}(x, p)$ is the upper boundary of $\Lambda_{i}^{F}(x, p)$, that is, $\partial \Lambda_{i}^{F}(x, p) \equiv\left\{y_{i} \in Q \mid y_{i} \in \Lambda_{i}^{F}(x, p)\right.$ and $\nexists z_{i} \in \Lambda_{i}^{F}(x, p)$ such that $\left.z_{i} \gg y_{i}\right\}$. For each $i$, let $\left(p^{i}, x_{i}^{i}\right)$ denote agent $i$ 's message. Finally, for each profile $m, u, x \in F(u)$ and $p \in \pi^{F}(x, u)$ define the sets

$N_{u}(m) \equiv\left\{k \in N \mid m_{k} \in T_{k}^{\gamma}(u, F(u))\right\}$ and $N_{u}(m ;(x, p)) \equiv\left\{k \in N_{u}(m) \mid m_{k}=\left(p, x_{k}\right)\right\}$.

With these preliminaries, we now define the outcome function $g$ of a $p-q$ mechanism $\gamma=(M, g)$ as follows. For each $m$,

Rule 1: If $m_{j}=\left(p, x_{j}\right)$ for each $j$ such that $x \equiv\left(x_{j}\right)_{j \in N} \in F\left(u^{\prime}\right)$ and $p \in \pi^{F}\left(x, u^{\prime}\right)$ for some $u^{\prime}$, and, moreover, $\sum x_{j}=\Omega$, then $g(m)=x$.

Rule 2: If $m_{j}=\left(p, x_{j}\right)$ with $x_{j} \neq 0$ for each $j, \sum x_{j} \neq \Omega$ and $I^{F}(p, x)=N$ where $x \equiv\left(x_{j}\right)_{j \in N}$, then $g(m)=\mathbf{z}(p, x)$.

Rule 3: If $m_{j}=\left(p, x_{j}\right)$ with $x_{j} \neq 0$ for each $j, 1 \leq \# I^{F}(p, x) \leq n-1$ where $x \equiv\left(x_{j}\right)_{j \in N}$, then $g(m)=\overline{\mathbf{z}}(p, x)$.

Rule 4: If, for some $i, m_{j}=\left(p, x_{j}\right)$ for all $j \neq i, m_{i}=\left(p^{i}, x_{i}\right)$ with $p \neq p^{i}$ and $x_{i} \neq 0$, and $i \in I^{F}(p, x)$ where $x \equiv\left(x_{j}\right)_{j \in N}$, then:

$$
g(m)= \begin{cases}\left(z_{i}\left(\left(p^{i}, x_{i}\right) ;\left(p, x_{-i}\right)\right), \mathbf{0}_{-i}\right) & \text { if } z_{i}\left(\left(p^{i}, x_{i}\right) ;\left(p, x_{-i}\right)\right) \neq 0 \\ \left(x_{i}, \mathbf{0}_{-i}\right) & \text { if } z_{i}\left(\left(p^{i}, x_{i}\right) ;\left(p, x_{-i}\right)\right)=0 \text { and } \\ & x_{i} \in \Lambda_{i}^{F}\left(\left(\Omega-\sum_{j \neq i} x_{j}, x_{-i}\right), p\right) \\ \left(\hat{x}_{i}, \mathbf{0}_{-i}\right) & \text { otherwise }\end{cases}
$$

where $\left\{\hat{x}_{i}\right\} \equiv \partial \Lambda_{i}^{F}\left(\left(\Omega-\sum_{j \neq i} x_{j}, x_{-i}\right), p\right) \bigcap\left\{y_{i} \in \mathbb{R}_{+}^{\ell} \mid \exists \alpha \in \mathbb{R}_{+}, y_{i}=\right.$ $\left.\alpha x_{i}\right\}$.

Rule 5: Otherwise,

Rule 5.1: If there is at least one agent $i$ who announces $x_{i}^{i}=0$ and there exists $\left(u^{\prime}, x^{\prime}, p^{\prime}\right)$, with $x^{\prime} \in F\left(u^{\prime}\right)$ and $p^{\prime} \in \pi^{F}\left(x^{\prime}, u^{\prime}\right)$, such that $N_{u^{\prime}}\left(m ;\left(x^{\prime}, p^{\prime}\right)\right)$ 
is non-empty and that $\# N_{u^{\prime}}\left(m ;\left(x^{\prime}, p^{\prime}\right)\right) \geq \# N_{u^{\prime \prime}}\left(m ;\left(x^{\prime \prime}, p^{\prime \prime}\right)\right)$ for all $\left(u^{\prime \prime}, x^{\prime \prime}, p^{\prime \prime}\right)$, with $x^{\prime \prime} \in F\left(u^{\prime \prime}\right)$ and $p^{\prime \prime} \in \pi^{F}\left(x^{\prime \prime}, u^{\prime \prime}\right),{ }^{18}$ then

$$
g(m)=\left(\frac{x_{1}^{\prime}}{(n+1)-\# N_{u^{\prime}}\left(m ;\left(x^{\prime}, p^{\prime}\right)\right)}, \ldots, \frac{x_{n}^{\prime}}{(n+1)-\# N_{u^{\prime}}\left(m ;\left(x^{\prime}, p^{\prime}\right)\right)}\right),
$$

Rule 5.2: Otherwise, $g(m)=\mathbf{0}$.

According to the proposed construction, $\gamma$ is a natural $p-q$ mechanism. Note that in Rule 4, $g_{i}(m)=\hat{x}_{i}$ if $p^{i} \in \partial \Delta$ and $x_{i}^{i} \in Q \backslash \Lambda_{i}^{F}\left(\left(\Omega-\sum_{j \neq i} x_{j}, x_{-i}\right), p\right)$, since $\left(p^{i}, x_{i}^{i}\right) \notin T_{i}^{\gamma}\left(u^{\prime} ; F\right)$ for all $u^{\prime}$. Thus, agent $i$ can realize any element of the set $\partial \Lambda_{i}^{F}\left(\left(\Omega-\sum_{j \neq i} x_{j}, x_{-i}\right), p\right)$ by a suitable choice of $\left(p^{i}, x_{i}^{i}\right)$.

Let $u$ be the true economy. Fix an arbitrary $H$. We shall show that $F(u)=N A\left(\gamma, \succcurlyeq^{u, H}\right)$. Since it is a routine exercise to prove $F(u) \subseteq$ $N A\left(\gamma, \succcurlyeq^{u, H}\right)$, we shall omit the proof here. Conversely, let $m \in N E\left(\gamma, \succcurlyeq^{u, H}\right)$. Note $m$ cannot corresponds to Rule 3, nor to Rule 4, as every agent who gets the zero bundle can induce Rule $\mathbf{5 . 1}$ which is a profitable deviation.

\section{Case 1: $m$ falls into Rule 1}

Then, $g(m)=x$. Note that each $i$ can induce Rule 4 and attain any bundle $\hat{x}_{i} \in \partial \Lambda_{i}^{F}(x, p)$ by announcing $m_{i}^{\prime}=\left(p^{\prime}, x_{i}^{\prime}\right)$, with $p^{\prime} \in \partial \Delta$ and $x_{i}^{\prime} \in Q \backslash \Lambda_{i}^{F}(x, p)$, such that $\left\{\hat{x}_{i}\right\} \equiv \partial \Lambda_{i}^{F}(x, p) \cap\left\{y_{i} \in \mathbb{R}_{+}^{\ell} \mid \exists \alpha \in \mathbb{R}_{+}\right.$such that $\left.y_{i}=\alpha x_{i}\right\}$. Thus, $\partial \Lambda_{i}^{F}(x, p) \subseteq g_{i}\left(M_{i}, m_{-i}\right) \subseteq L\left(x_{i}, u_{i}\right)$ for each $i$. By the strong monotonicity of $u_{i}, \Lambda_{i}^{F}(x, p) \subseteq L\left(x_{i}, u_{i}\right)$ for each $i$.

If $i \in H, m_{i} \notin T_{i}^{\gamma}(u ; F)$, and there exists $m_{i}^{\prime}=\left(p^{\prime}, x_{i}^{\prime}\right) \in T_{i}^{\gamma}(u ; F)$ such that $g_{i}\left(m_{i}^{\prime}, m_{-i}\right) \in \partial L\left(x_{i}, u_{i}\right)$, then $\left(\left(m_{i}^{\prime}, m_{-i}\right), m\right) \in \succ_{i}^{u}$, which contradicts $m \in N E\left(\gamma, \succcurlyeq^{u, H}\right)$. Therefore, for each $i \in H, m_{i} \notin T_{i}^{\gamma}(u ; F)$ implies $g_{i}\left(\left(p^{\prime}, x_{i}^{\prime}\right), m_{-i}\right)=z_{i}\left(\left(p^{\prime}, x_{i}^{\prime}\right) ;\left(p, x_{-i}\right)\right) \notin \partial L\left(x_{i}, u_{i}\right)$ for any $\left(p^{\prime}, x_{i}^{\prime}\right) \in$ $T_{i}^{\gamma}(u ; F)$. By MP-SH(iv), $x \in F(u)$.

\section{Case 2: $m$ falls into Rule 2}

Then, $g(m)=\mathbf{z}(p, x)$. If $\sum \mathbf{z}_{i}(p, x)<\Omega$, since $F$ is efficient, $\mathbf{z}(p, x) \notin$ $F(u)$. By the same arguments as in Case 1, $\Lambda_{i}^{F}\left(\left(\Omega-\sum_{j \neq i} x_{j}, x_{-i}\right), p\right) \subseteq$

\footnotetext{
${ }^{18}$ If there is more than one of set $N_{u^{\prime}}\left(m ;\left(x^{\prime}, p^{\prime}\right)\right)$ having the same cardinaltiy, then fix any one of the sets.
} 
$L\left(\mathbf{z}_{i}(p, x), u_{i}\right)$ for each $i$. Then, by $\mathbf{M P}-\mathbf{S H}($ iv), there is $i \in H$ with $m_{i}^{\prime}=\left(p^{\prime}, x_{i}^{\prime}\right) \in T_{i}^{\gamma}(u ; F)$ such that $g_{i}\left(m_{i}^{\prime}, m_{-i}\right)=z_{i}\left(\left(p^{\prime}, x_{i}^{\prime}\right) ;\left(p, x_{-i}\right)\right) \in$ $\partial L\left(\mathbf{z}_{i}(p, x)_{i}, u_{i}\right)$, which contradicts $m \in N E\left(\gamma, \succcurlyeq^{u, H}\right)$. Thus, $\sum \mathbf{z}_{i}(p, x)=$ $\Omega$. Following the same arguments as in Case $\mathbf{1}, \mathbf{z}(p, x) \in F(u)$ by MP-SH.

\section{Case 3: $m$ falls into Rule 5}

Since agents in $H$ have strong intrinsic preferences for honesty and moreover, agents respond primarily to material incentives, it is easy to see that $m$ cannot correspond to Rule 5.2. ${ }^{19}$ We show that $m$ cannot correspond to Rule 5.1 either.

Suppose that $m$ falls into Rule 5.1. Then,

$$
g_{k}(m)=\frac{x_{k}^{\prime}}{(n+1)-\# N_{u^{\prime}}\left(m ;\left(x^{\prime}, p^{\prime}\right)\right)} \text { for each } k \text {. }
$$

Suppose that $m$ is such that there are at least two agents, say $i$ and $i^{\prime}$, who announce the zero vector 0 . By changing $m_{i}$ into $m_{i}^{\prime}=\left(p^{\prime}, x_{i}^{\prime}\right)$ such that $i \in N_{u^{\prime}}\left(\left(m_{-i}, m_{i}^{\prime}\right) ;\left(x^{\prime}, p^{\prime}\right)\right), i$ induces Rule $\mathbf{5 . 1}$ and obtains

$$
g_{i}\left(m_{-i}, m_{i}^{\prime}\right)=\frac{x_{i}^{\prime}}{(n+1)-\# N_{u^{\prime}}\left(\left(m_{-i}, m_{i}^{\prime}\right) ;\left(x^{\prime}, p^{\prime}\right)\right)}>g_{i}(m),
$$

\footnotetext{
${ }^{19}$ Assume, to the contrary, that $m \in N E\left(\gamma, \succcurlyeq^{u, H}\right)$ falls into Rule 5.2. Then, $g_{i}(m)=0$ and $x_{i}^{i} \neq 0$ for each $i$. Moreover, for each $i \in H, m_{i} \in T_{i}^{\gamma}(u ; F)$, otherwise, each $i \in H$ with $m_{i} \notin T_{i}^{\gamma}(u ; F)$ can switch $m_{i}$ to $m_{i}^{\prime} \in T_{i}^{\gamma}(u ; F)$ and obtain $g_{i}\left(m_{i}^{\prime}, m_{-i}\right) \geq g_{i}(m)$, which yields a contradiction. Furthermore, any $i \notin H$ if $H \neq N$, or else any $i$, can switch $m_{i}=\left(p^{i}, x_{i}^{i}\right)$ to $m_{i}^{\prime}=\left(p^{i}, 0\right)$. Since in $\left(m_{i}^{\prime}, m_{-i}\right)$ there are agents who report strongly truthful messages for $u$, it follows that for some $x \in F(u)$ and some $p \in \pi^{F}(x, u)$, the set $N_{u}\left(\left(m_{i}^{\prime}, m_{-i}\right) ;(x, p)\right)$ is non-empty. Among the pairs $(x, p)$ in $F(u) \times \pi^{F}(x, u)$, take any pair $\left(x^{0}, p^{0}\right)$ such that $\# N_{u}\left(\left(m_{i}^{\prime}, m_{-i}\right) ;\left(x^{0}, p^{0}\right)\right) \geq \# N_{u}\left(\left(m_{i}^{\prime}, m_{-i}\right) ;(x, p)\right)$ for all pairs $(x, p)$ in $F(u) \times$ $\pi^{F}(x, u)$. Note that $N_{u}\left(\left(m_{i}^{\prime}, m_{-i}\right) ;\left(x^{0}, p^{0}\right)\right) \neq N$. If $\# N_{u}\left(\left(m_{i}^{\prime}, m_{-i}\right) ;\left(x^{0}, p^{0}\right)\right) \geq$ $\# N_{u^{*}}\left(\left(m_{i}^{\prime}, m_{-i}\right) ;\left(x^{*}, p^{*}\right)\right)$ for each $\left(u^{*}, x^{*}, p^{*}\right)$ in $U_{N} \times F\left(u^{*}\right) \times \pi^{F}\left(x^{*}, u^{*}\right)$, then $\left(m_{i}^{\prime}, m_{-i}\right)$ falls into Rule 5.1 and agent $i$ obtains $g_{i}\left(m_{i}^{\prime}, m_{-i}\right)>g_{i}(m)$, which produces a contradiction. Otherwise, suppose that there exists a triple $\left(u^{1}, x^{1}, p^{1}\right)$, with $x^{1} \in F\left(u^{1}\right)$ and $p^{1} \in \pi^{F}\left(x^{1}, u^{1}\right)$, such that $\# N_{u^{1}}\left(\left(m_{i}^{\prime}, m_{-i}\right) ;\left(x^{1}, p^{1}\right)\right)>\# N_{u}\left(\left(m_{i}^{\prime}, m_{-i}\right) ;\left(x^{0}, p^{0}\right)\right)$. If $\# N_{u^{1}}\left(\left(m_{i}^{\prime}, m_{-i}\right) ;\left(x^{1}, p^{1}\right)\right) \geq \# N_{u^{*}}\left(\left(m_{i}^{\prime}, m_{-i}\right) ;\left(x^{*}, p^{*}\right)\right)$ for each $\left(u^{*}, x^{*}, p^{*}\right)$ in $U_{N} \times$ $F\left(u^{*}\right) \times \pi^{F}\left(x^{*}, u^{*}\right)$, then $\left(m_{i}^{\prime}, m_{-i}\right)$ falls into Rule $\mathbf{5 . 1}$ and agent $i$ obtains a profitable deviation, which is a contradiction. And so on. After a finite number $l$ of iterations, with $\# N_{u}\left(\left(m_{i}^{\prime}, m_{-i}\right) ;\left(x^{0}, p^{0}\right)\right) \leq l<n$, we can find a triple $\left(u^{l}, x^{l}, p^{l}\right)$ such that $\# N_{u^{l}}\left(\left(m_{i}^{\prime}, m_{-i}\right) ;\left(x^{l}, p^{l}\right)\right) \geq \# N_{u^{*}}\left(\left(m_{i}^{\prime}, m_{-i}\right) ;\left(x^{*}, p^{*}\right)\right)$ for each $\left(u^{*}, x^{*}, p^{*}\right)$ in $U_{N} \times F\left(u^{*}\right) \times \pi^{F}\left(x^{*}, u^{*}\right)$. Therefore, $\left(m_{i}^{\prime}, m_{-i}\right)$ falls into Rule 5.1 and agent $i$ obtains a profitable deviation, which is a contradiction.
} 
thereby yielding a contradiction. Therefore, $m$ is such that there is only one agent $i$ who announces $x_{i}^{i}=0$. Moreover, suppose that $m$ is such that $\# N_{u^{\prime}}\left(m ;\left(x^{\prime}, p^{\prime}\right)\right)<n-1$. Take an arbitrary $j$ such that $j \neq i$ and $j \notin N_{u^{\prime}}\left(m ;\left(x^{\prime}, p^{\prime}\right)\right)$. By changing $m_{j}$ into $m_{j}^{\prime}=\left(p^{\prime}, x_{j}^{\prime}\right)$ such that $j \in N_{u^{\prime}}\left(\left(m_{-j}, m_{j}^{\prime}\right) ;\left(x^{\prime}, p^{\prime}\right)\right), j$ induces Rule $\mathbf{5 . 1}$ and obtains

$$
g_{j}\left(m_{-j}, m_{j}^{\prime}\right)=\frac{x_{j}^{\prime}}{(n+1)-\# N_{u^{\prime}}\left(\left(m_{-j}, m_{j}^{\prime}\right) ;\left(x^{\prime}, p^{\prime}\right)\right)}>g_{j}(m),
$$

which produces a contradiction. Therefore, $m$ is such that there is only one agent $i$ who announces $x_{i}^{i}=0$, the set $N_{u^{\prime}}\left(m ;\left(x^{\prime}, p^{\prime}\right)\right)$ has cardinality equal to $n-1$ and

$$
g(m)=\left(\frac{x_{1}^{\prime}}{2}, \ldots, \frac{x_{n}^{\prime}}{2}\right) .
$$

By changing $m_{i}$ into $m_{i}^{\prime}=\left(p^{\prime}, x_{i}^{\prime}\right)$ with $i \in N_{u^{\prime}}\left(\left(m_{-i}, m_{i}^{\prime}\right) ;\left(x^{\prime}, p^{\prime}\right)\right), i$ induces Rule 1 and obtains $g_{i}\left(m_{-i}, m_{i}^{\prime}\right)=x_{i}^{\prime}$, which again yields a contradiction.

Since $H$ is arbitrary, it follows that $F(u)=N A\left(\gamma, \succcurlyeq^{u, H}\right)$ for each $H$. Finally, since $u$ is arbitrary, the statement follows.

Proof of Theorem 2. Suppose all the hypotheses of the statement hold. For the sake of notational parsimony, let $\underbrace{U \times \ldots \times U}_{n \text { times }} \equiv \bar{U}$.

Claim 1 Let $(u, x) \in \bar{U} \times W(u)$ be given. The set $\Pi^{W}(x, u)$ in (3) is never empty. Furthermore, it consists solely of Walrasian equilibrium prices for $x$, that is, if $p \in \Pi^{W}(x, u)$, then $p \cdot x_{i}=p \cdot \omega_{i}$ for each $i$.

Proof of Claim 1. Note that since $W$ is defined on $\bar{U}$, if $\left(x, p^{W}\right)$ is a Walrasian equilibrium for $u$, then the set $W_{!}^{-1}\left(x, p^{W}\right)$ defined in (2) is never empty. From this observation, it can easily be seen that the set $\Pi^{W}(x, u)$ is non-empty. To see that $\Pi^{W}(x, u)$ consists solely of Walrasian equilibrium prices for $x$, take an arbitrary $p \in \Pi(x, u)$ such that for some $i, p \cdot x_{i} \neq p \cdot \omega_{i}$. Then, $(x, p)$ is not a Walrasian equilibrium for any $u^{\prime}$ in $\bar{U}$. It follows that $W_{!}^{-1}(x, p)$ is empty and so $p$ is not in $\Pi^{W}(x, u)$. Since $p$ is arbitrary, the statement follows.

Since the pair $(u, x) \in \bar{U} \times W(u)$ in Claim 1 is arbitrary, the sets $\pi^{W}(x, u)$ possesses the following property:

$$
\pi^{W}(x, u)=\Pi^{W}(x, u) \text { for each }(u, x) \in \bar{U} \times W(u),
$$


and for each $p \in \pi^{W}(x, u)$ and each $i$, the set $\Lambda_{i}^{W}(x, p)$ coincides with the constrained Walrasian budget set ${ }^{20}$ :

$$
\Lambda_{i}^{W}(x, p) \equiv \bigcap_{u^{\prime} \in W_{!}^{-1}(x, p)} L\left(x_{i}, u_{i}^{\prime}\right)=\left\{y_{i} \in Q \mid p \cdot y_{i} \leq p \cdot \omega_{i}\right\}
$$

Next, we show that $W$ satisfies MP-SH. For, let $H$ and $(p, x) \in \Delta \times Q^{n}$ be given. Since MP-SH contains four parts, we proceed by breaking the proof into a series of claims.

Claim $2 W$ satisfies $\boldsymbol{M P}-\boldsymbol{S H}(i)$.

Proof of Claim 2. Suppose $(p, x)$ is such that $I^{W}(p, x)=N$. Define the allocation $\mathbf{z}(p, x)$ as follows:

$$
\mathbf{z}(p, x)= \begin{cases}x & \text { if } \sum x_{i}=\Omega \\ \mathbf{0} & \text { otherwise }\end{cases}
$$

It is clear that $\mathbf{z}_{i}(p, x) \in \Lambda_{i}^{W}\left(\left(\Omega-\sum_{j \neq i} x_{j}, x_{-i}\right), p\right)$ for each $i$. Therefore, $W$ satisfies $\mathbf{M P}-\mathbf{S H}(\mathrm{i})$.

Claim $3 W$ satisfies $\boldsymbol{M P}$-SH(ii).

Proof of Claim 3. Suppose that $1 \leq \# I^{W}(p, x) \leq n-1$. Define the allocation $\overline{\mathbf{z}}(p, x)$ as follows: For each $i$,

$$
\overline{\mathbf{z}}_{i}(p, x)=0 .
$$

It follows easily from the above definition that $\sum \overline{\mathbf{z}}_{i}(p, x) \leq \Omega$ and that $\overline{\mathbf{z}}_{i}(p, x) \in \Lambda_{i}^{W}\left(\left(\Omega-\sum_{j \neq i} x_{j}, x_{-i}\right), p\right)$ for each $i$. We conclude that $W$ satisfies MP-SH(ii).

Claim 4 W satisfies $\boldsymbol{M P}-\boldsymbol{S H}($ iii).

\footnotetext{
${ }^{20}$ The constrained Walrasian budget set is the intersection of the standard budget set (defined by $p$ and $\omega_{i}$ ) with $Q$.
} 
Proof of Claim 4. Define the functions $\mathbf{z}$ and $\overline{\mathbf{z}}$ as they are defined in the proofs of Claims 2-3. Fix an arbitrary $i$. Define the real-valued function $z_{i}\left(\cdot ; p, x_{-i}\right)$ as follows: For each $\left(p^{\prime}, x_{i}^{\prime}\right)$,

1. If $\left(p^{\prime}, x_{i}^{\prime}\right)$ is strongly truthful for some $u^{\prime}$ and $i \in I^{W}\left(p,\left(x_{i}^{\prime}, x_{-i}\right)\right)$, then

$$
z_{i}\left(\left(p^{\prime}, x_{i}^{\prime}\right) ;\left(p, x_{-i}\right)\right)=\left\{\begin{array}{cl}
\Omega-\sum_{j \neq i} x_{j} & \text { if } p^{\prime} \neq p \\
\mathbf{z}_{i}(p, x) & \text { if }\left(p^{\prime}, x_{i}^{\prime}\right)=\left(p, x_{i}\right) \text { and } I^{W}(p, x)=N \\
\overline{\mathbf{z}}_{i}(p, x) & \text { if } p^{\prime}=p \text { and } 1 \leq \# I^{W}\left(p,\left(x_{i}^{\prime}, x_{-i}\right)\right) \leq n-1
\end{array}\right.
$$

2. Otherwise, $z_{i}\left(\left(p^{\prime}, x_{i}^{\prime}\right) ;\left(p, x_{-i}\right)\right)=0$.

Given the definitions in (9)-(10) and given the above definition of $z_{i}\left(\cdot ; p, x_{-i}\right)$, one easily checks that $W$ satisfies $\mathbf{M P - S H}($ iii).

Claim 5 W satisfies $\boldsymbol{M P}-\boldsymbol{S H}(i v)$.

Proof of Claim 5. Define the functions $\mathbf{z}, \overline{\mathbf{z}}$ and $z_{i}$ as they are defined in the proofs of Claims 2-4. Fix an arbitrary $u$. Suppose that $I^{W}(p, x)=N$, $\Lambda_{k}^{W}\left(\left(\Omega-\sum_{j \neq k} x_{j}, x_{-k}\right), p\right) \subseteq L\left(\mathbf{z}_{k}(p, x), u_{k}\right)$ for each $k$, and $\mathbf{z}(p, x) \notin$ $W(u)$. Given the definition of $\mathbf{z}(p, x)$ in $(9)$, the only case to consider is $\sum x_{i}=\Omega$. Then, in this case, $\mathbf{z}(p, x)=x$, and so, by definitions, $(x, p)$ is a Walrasian equilibrium and $p \in \pi^{W}\left(x, u^{\prime}\right)$ for some $u^{\prime}$.

Moreover, since $\Lambda_{k}^{W}(x, p) \subseteq L\left(x, u_{k}\right)$ for each $k, \mathbf{z}(p, x)=x \in W\left(u^{\prime}\right) \backslash W(u)$ implies that there is an agent $k$ for whom $x_{k} \notin \arg \max _{y_{i} \in \mathbb{R}_{+}^{\ell}: p \cdot y_{k} \leq p \cdot \omega_{k}} u_{k}\left(y_{k}\right)$. However, since $x_{k} \in \arg \max _{y_{i} \in Q: p \cdot y_{k} \leq p \cdot \omega_{k}} u_{k}\left(y_{k}\right)$ by $\Lambda_{k}^{W}(x, p) \subseteq L\left(x, u_{k}\right)$, $\left(\arg \max _{y_{i} \in \mathbb{R}_{+}^{\ell}: p \cdot y_{k} \leq p \cdot \omega_{k}} u_{k}\left(y_{k}\right)\right) \cap Q=\varnothing$ holds, which suggests that $p$ cannot be a Walrasian equilibrium price vector at $u$. Thus, for any $i \in H$, the pair $\left(p, x_{i}\right)$ is not strongly truthful for $u$. This verifies MP-SH(iv.a). Let $\left(p^{\prime}, x_{i}^{\prime}\right)$ be any strongly truthful pair for $u$. Then, $p^{\prime} \neq p$. By $I^{W}(p, x)=$ $N, i \in I^{W}\left(p,\left(x_{i}^{\prime}, x_{-i}\right)\right)$. By definition of $z_{i}\left(\cdot ;\left(p, x_{-i}\right)\right)$ in (11), we have $z_{i}\left(\left(p^{\prime}, x_{i}^{\prime}\right) ;\left(p, x_{-i}\right)\right)=x_{i}$, thus $W$ satisfies MP-SH(iv.b).

The proof concludes by observing that the set $H$ and the pair $(p, x)$ were arbitrarily taken. 


\section{References}

[1] Corchón, L., Herrero, C., 2004. A decent proposal. Spanish Economic Review, 6, 107-125.

[2] Dutta, B., Sen, A., 2012. Nash implementation with partially honest individuals. Games and Economic Behavior, 74, 154-169.

[3] Dutta, B., Sen, A., Vohra, R., 1995. Nash implementation through elementary mechanisms in economic environments. Economic Design, 1, 173-204.

[4] Eliaz, K., 2002. Fault-tolerant implementation. Review of Economic Studies, 69, 589-610.

[5] Glazer, J., Rubinstein, A., 1998. Motives and implementation: On the design of mechanisms to elicit opinions. Journal of Economic Theory, $79,157-173$.

[6] Hurwicz, L., 1978. On the interaction between information and incentives in organizations, in Krippendorff, K. Communication and Control in Society (Scientific Publishers, Inc., New York), 123-147.

[7] Hurwicz, L., Maskin, E., and Postlewaite A., 1995. Feasible Nash implementation of social choice rules when the designer does not know endowments or production sets, in Ledyard, J. (ed.), The economics of informational decentralization: Complexity, efficiency, and stability, Essays in Honor of Stanley Reiter, pp. 367-433. Amsterdam: Kluwer Academic Publishers.

[8] Jackson, M., 1992. Implementation in undominated strategies: A look at bounded mechanisms. Review of Economic Studies, 59, 757-775.

[9] Kartik, N., Tercieux, O., 2012. Implementation with evidence. Theoretical Economics, 7, 323-355.

[10] Kartik, N., Tercieux, O., Holden, R., 2014. Simple mechanisms and prefereces for honesty. Games and Economic Behavior, 83, 284-290.

[11] Lombardi, M., Yoshihara, N., 2013a. A full characterization of Nash implementation with strategy space reduction. Economic Theory, 54, 131-151. 
[12] Lombardi, M., Yoshihara, N., 2013b. Natural implementation with partially honest agents. IER Discussion Paper Series A, No.561, The Institute of Economic Research, Hitotsubashi University.

[13] Maskin, E., 1999. Nash equilibrium and welfare optimality. Review of Economic Studies, 66, 23-38.

[14] Matsushima, H., 2008a. Role of honesty in full implementation. Journal of Economic Theory, 139, 353-359.

[15] Matsushima, H., 2008b. Behavioral aspects of implementation theory. Economics Letters, 100, 161-164.

[16] Otani, Y., Sicilian, J., 1982. Equilibrium of Walras preference games. Journal of Economic Theory, 27, 47-68.

[17] Saijo, T., Tatamitani, Y., Yamato, T., 1996. Toward natural implementation. International Economic Review, 37, 949-980.

[18] Saijo, T., Tatamitani, Y., Yamato, T., 1999. Characterizing natural implementability: The fair and Walrasian correspondences. Games and Economic Behavior, 28, 271-293.

[19] Sjöström, T., 1996. Implementation by demand mechanisms. Economic Design, 1, 343-354.

[20] Thomson, W., 1984. The manipulability of resource allocation mechanisms. Review of Economic Studies, 51, 447-460. 